\title{
Hydraulic resistance of a plant root to water-uptake: a slender-body theory
}

\author{
Kang Ping Chen \\ School for Engineering of Matter, Transport and Energy \\ Arizona State University, Tempe, AZ 85287-6106, USA
}

\begin{abstract}
A slender-body theory for calculating the hydraulic resistance of a single plant root is developed. The work provides an in-depth discussion on the procedure and the assumptions involved in calculating a root's internal hydraulic resistance as well as the physical and the mathematical aspects of the external three-dimensional flow around the tip of a root in a saturated soil and how this flow pattern enhances uptake and reduces hydraulic resistance. Analytical solutions for the flux density distribution on the stele-cortex interface, local wateruptake profile inside the stele core, the overall water-uptake at the base of the stele, and the total hydraulic resistance of a root are obtained in the slender-body limit. It is shown that a key parameter controlling a root's hydraulic resistance is the dimensionless axial conductivity in the stele, which depends on the permeabilities of the stele and the cortex as well as the root's radial and axial dimensions. Three-dimensional tip effect reduces a root's hydraulic resistance by as much as $36 \%$ when compared to the radial flow theory of Landsberg \& Fowkes. In addition, the total hydraulic resistance cannot be generally decomposed into the direct sum of a radial resistance and an axial resistance.
\end{abstract}

Email: k.p.chen@asu.edu; Tel: (480)965-0907; Fax: (480)727-9321 


\section{Introduction}

Water-uptake from soil by a plant's root system is a problem of vital interest to plant biology, agriculture and ecology (Steudle \& Peterson, 1998; Steudle, 2000; Raats, 2007; Roose \& Schnepf, 2008; Stroock et al., 2014). Significant efforts have been made in the past five decades to quantitatively model water-uptake by a plant's root system, spanning from the mesoscopic single-root scale to the entire root system at the macroscopic scale (Philip, 1957; Gardner, 1960; Cowan, 1965; Landsberg \& Fowkes, 1978; Molz, 1981; Passioura, 1988; Steudle, 2000; Raats, 2007; Roose \& Schnepf, 2008; Stroock et al., 2014). Various approaches have been used, from considering water flow inside the root structure only (Doussan et al., 1998) to considering water flow in the soil only (Gardner, 1960; Clausnitzer \& Hopmans, 1994), and to the ones that considering simultaneous movement of water in the soil and the root system (Roose \& Fowler, 2004; Roose \& Schnepf, 2008). At the macroscopic root system level, water-uptake by a plant root is coupled to water transport in an unsaturated soil by the Richard's equation (Raats, 2007)

$$
\frac{\partial \theta}{\partial t}=\nabla \cdot[K \nabla p]-S
$$

where $\theta$ is the volumetric water content (or relative water saturation; moisture content); $K=K(\theta)$ is the conductivity in the soil which depends on the local water content; $p$ is the water

pressure in the soil pores which is also linked to the local water content, $p=p(\theta)$; and $S$ is a volumetric sink term representing root water-uptake. Typical relations for $K(\theta)$ and $p(\theta)$ are given by van Genuchten (1980), for example. The sink term $S$ is also called the water extraction function (Molz, 1981). Since the Richard's equation (1) describes water transport in the soil at a much larger length scale than the mesoscopic scale of a single rootlet, the sink term $S$ is an uptake averaged over a large number of rootlets (Raats, 2007). $S$ is therefore directly linked to the water-uptake by a single root calculated at the mesoscopic level. Molz (1981) provided a list 
of various empirical expressions and models for this sink term up to 1981. Raats (2007) discussed in detail a procedure of obtaining the sink term $S$ from the water flow into an individual root, i.e. from a mesoscopic single root model. Volumetric rate of uptake expressed as a sink $S$ in the Richard's equation is at the heart of all macroscopic models; and it must be derived from an analysis of the uptake at the mesoscopic single root scale.

At the mesoscopic scale, a single root has been historically modeled geometrically as a long cylinder with a constant radius, drawing water from the soil in a cylindrical shell (Philip, 1957; Gardner, 1960; Cowan, 1965; Landsberg \& Fowkes, 1978; Hainsworth \& Aylmore, 1986; Raats, 2007). Inside the root, the water flow in the stele is predominantly longitudinal, and it is controlled by the flow through the xylem tubes (Fig. 1). In the root's cortex and in the soil, water has been modeled to flow only in the radial direction, perpendicular to the cylinder's axis of symmetry. Once the uptake is computed from this simple model for a unit length of the cylindrical root, the volumetric sink term $S$ in the macroscopic Richard's equation is then computed by multiplying the single root uptake by the so-called root length density (Roose \& Folwer, 2004; Raats, 2007; Roose \& Schnepf, 2008). All of the models for the volumetric sink S discussed in Molz (1981) and Raats (2007) are in fact variations of the original Gardner's twodimensional radial flow model. However, a two-dimensional radial flow occurs only when the cylinder is infinitely long. Therefore, this widely used model has a serious limitation since all roots have a finite length and the flow around the end of a finite-length cylinder (root) is always three-dimensional. An outcome of neglecting this three-dimensional tip effect is the underestimation of the water flux density on the root-soil surface, which in turn results in an underestimation of the water-uptake by a single root. This point of view is supported by the 
consideration of the water flow pattern in the soil near a finite-length root shown in Fig. 2 (Chen, 2015). In this example, the finite-length root is geometrically modeled as a slender semi-prolatespheroid; water in the soil is assumed to be fully saturated and obeys Darcy's law; and the pressure on the root-soil surface is a constant. The mathematical solution for the flow in the soil shown in Fig. 2 is described in Section 4. In Fig. 2, the streamlines of water flow towards the finite-length root clearly show a converging pattern near the tip region of the root. This flow pattern indicates that a significant amount of water enters the root surface from the near tip region. A similar converging flow pattern also occurs for an unsaturated soil, as the differential operator governing water flow in an unsaturated soil is essentially the same as that for a saturated soil (i.e. Laplacian). Modifications caused by the saturation function do not alter the nature of the differential operator, which controls the process of outward diffusion of the fluid pressure drawdown through the soil. In other words, flow convergence to the root tip is a general phenomenon in both saturated and unsaturated soils. This converging flow pattern, however, cannot be captured by the infinitely long cylinder model which predicts two-dimensional flows with streamlines perpendicular to the root axis; or any other model that ignores the threedimensional nature of the flow around a root tip.

On the other hand, an argument against the tip effect could be made based on the finding of the "hydraulically isolated" zone near the tip of a root by Frensch \& Steudle (1989). Frensch \& Steudle (1989) experimentally found that the axial conductivity of the xylem decreases from a constant value to zero within a short distance to the tip. The cone-shaped tip region requires the radius of all xylem vessels to shrink to zero in the region. Therefore, the anatomy of the root makes the tip region not “functional" for water uptake; and a root's water uptake is accomplished 
only by the portion of the root behind the tip region. This line of thinking seems to justify modeling a root by a cylinder with a sealed end, with the flow from the soil to the root and the flow from the cortex annulus to the stele core being purely radial, corresponding to the models of Gardner (1960) and Landsberg \& Fowkes (1978), respectively. From a fluid mechanics point of view, however, the converging flow pattern shown in Fig. 2 must persist for a long but finitelength cylinder even when the end is sealed. This is due to the fact that the gradient of the water pressure in the soil becomes very large near the end of the slender cylinder, which induces very large local water flux density according to Darcy's law. This is a phenomenon common to low Reynolds number flow and Darcy flow near a corner (Chen et al, 2013; Jin et al. 2015; Chen 2015). Thus, even if direct entry through the end surface (or nose) of the cylinder is blocked because of the root anatomy near the tip, substantial amount of water still enters the root from the tip region through the side surface. This focusing effect forms a convergent flow pattern near the end of the root, not too different from the flow pattern for an unsealed end shown in Fig. 2. Flow focusing is also one of the physical principles behind the effectiveness of using a hydraulic fracture to increase oil and gas production (Chen et al., 2013; Jin et al., 2015).

The "hydraulically isolated" tip zone argument discussed above is also countered by the radial conductivity measurements made by Melchior \& Steudle (1993) in onion roots. Melchior \& Steudle (1993) found that while a root's axial conductivity increases from the tip region to the basal region, the radial conductivity in the tip region is at least five-fold higher than that in the basal region. It is then not unreasonable to argue that such a high radial conductivity near the tip region will attract water stored in the soil to flow towards the tip region instead of the basal region, creating large local flux densities at the root-soil surface near the tip. This is consistent 
with the flow focusing phenomenon discussed above. Thus, the radial conductivity measurements of Melchior \& Steudle (1993) implicitly support the converging flow pattern near the tip.

The physics-based reasoning that water flow in the soil converges to the tip region is further supported by the mathematics of Darcy flow towards a slender-body. In this discussion, we assume the worst case scenario that the "hydraulically-dead" zone near the root tip causes the tip region to be sealed, which is characterized by the non-penetration condition of $\partial p$ / $\partial n=0$ on the sealed part of the root-soil surface, with $p$ being the fluid pressure and $\mathbf{n}$ the unit outward normal on the root-soil surface (Fig. 3). The rest of the root-soil surface is porous, characterized by the constant pressure (or potential) boundary condition (Fig. 3 (a)). In the slender-body limit, a model first developed for aerodynamics of thin airfoils (Batchelor, 1967), the root is approximated as having a zero radius, “collapsing” mathematically to the z-axis (Fig. 3 (b)). The normal $\mathbf{n}$ is then in the radial direction, perpendicular to the $\mathbf{z}$-axis. Since the flow in the soil is symmetric about the z-axis, the boundary condition along the portion of the z-axis outside the root (i.e. in the soil) is also $\partial p / \partial n=0$. Thus, the sealed portion of the root tip can be "merged/combined" mathematically to the z-axis outside the tip because they obey the same boundary condition. In other words, in the slender-body limit, the effect of "a hydraulically dead tip zone” is to shorten the original root to a root with a shorter, equivalent length (Fig. 3). In the slender-body theory, the equivalent root is part of the z-axis; and at the tip of this equivalent root, there is a discontinuity in the boundary condition: for the equivalent root, the potential is prescribed; while for the z-axis outside the equivalent root, the non-penetration condition (or symmetry condition) is imposed. It is well-known that this kind of discontinuity in the boundary 
condition induces an integrable singularity in the gradient of the potential (i.e. pressure gradient) for the potential problem (or the diffusion problem) (Michael, 1958; Moffatt, 1964; Richardson, 1970; Trogdon \& Joseph, 1980; Sprittles \& Shikhmurzaev 2011). Thus, the Darcy velocity in the soil becomes unbounded at the tip of the equivalent root. In other words, the water flux density on the root-soil surface is singular at the tip of the equivalent root. This singular water flux density at the tip is consistent with the underlying physics discussed above: the flow in the soil converges to the tip region even when the end of the root is physically impermeable (sealed). Also of importance is that the existence of this singularity is independent of the size of the "hydraulically isolated tip zone”, as long as the root is thin and long so that the slender-body theory can be applied.

At present there is no flow visualization experiment to directly confirm the converging flow pattern discussed above. Direct measurement of the water flux density on the root-soil surface near the tip zone is difficult. While the local water-uptake at the tip is always zero, it does not imply that the water flux density on the root-soil surface is also zero, as the water flux density is the longitudinal gradient (gradient in the z-direction) of the local water uptake (Section 5). This conceptual difference has caused confusions in the literature previously. To show that there is a singular water flux density near the tip, one needs to accurately measure the rapid change in the local water uptake in the immediate vicinity of the tip. The lack of direct experimental measurements to confirm the tip effect, however, should not be construed as an "evidence" against the flow focusing phenomenon, as this phenomenon is genuine and strongly supported by the flow physics and mathematics as well as the radial conductivity measurement of Melchior \& Steudle (1993). 
The three-dimensional tip effect enhances a single root's water uptake and it must be reflected in the macroscopic scale model such as the Richard's equation (1). One way to incorporate this effect to improve the accuracy of the sink term $S$ in the macroscopic scale Richard's equation is to model the sink term $S$ as

$$
S=N(\mathbf{x}) A_{0} \frac{p(\theta)-p_{r}(\mathbf{x})}{R}
$$

where $N(\mathbf{x})$ is the root density distribution (number of root per unit soil volume); $A_{0}$ is the average of the root base cross-sectional area for the roots in this local volume; $R$ is the single root hydraulic resistance; $p(\theta)-p_{r}(\mathbf{x})$ is the difference between the water pressure in the soil and the water pressure in the root; and $A_{0}\left(p(\theta)-p_{r}(\mathbf{x})\right) / R$ is the water-uptake by a single root. The hydraulic resistance $R$ is the ratio between the pressure drop ("voltage”) from the root-soil surface to the root base and the water-uptake rate per unit cross-sectional area at the base (“electric current-intensity”). The hydraulic resistance is regarded as a property of the root which depends on the root's internal structure. Thus, the three-dimensional tip effect that enhances a root's water uptake is equivalent to a reduction in a single root's hydraulic resistance $R$.

To find the hydraulic resistance of a single root, we impose a constant pressure drop between the root surface and the base of the stele, and solve for the water volumetric flow rate though the stele core. This is the same method used to find the thermal resistance of a structure in heat conduction and the electric resistance in an electric network. At first glance, a root's internal hydraulic resistance does not seem to be directly related to the water flow pattern in the soil, as it 
is determined by the internal hydraulics of the root. However, the method for calculating the hydraulic resistance implicitly invokes two assumptions:

(i) There is always sufficient water supply from the soil to the root, implying that the soil is always saturated with water with no limit on its availability. Only under this condition that the measured/computed resistance is a true property of the structure, since the resistance obtained in this manner is solely due to the structure itself;

(ii) The flow pattern inside the root used to compute the hydraulic resistance must be hydrodynamically compatible with the flow pattern in the soil.

It must be emphasized that to study the intrinsic resistance of a structure, we must set up a problem that is not "supply-limited". The same methodology and assumptions are used in calculating thermal resistance and electric resistance, with the only difference being that different "matters" or "physical quantities" are transported. It is through the fluid mechanics requirements (i) and (ii) that the converging flow pattern in the soil near the root tip influences the flow pattern inside the root, thus a root's internal hydraulic resistance. Therefore, if the external flow in the soil creates high flux denisties near the tip of the root, the flow inside the root in the vicinity of the tip must also exhibit similar high local flux densities on the stele-cortex interface.

The objective of the present study is to provide a more accurate calculation of the internal hydraulic resistance of a single root $R$ by taking into account the three-dimensional end effect. At present, the standard mathematical model on the internal hydraulic resistance of a single root is that due to Landsberg \& Fowkes (1978). In the work of Landsberg \& Fowkes, the bundle of xylem tubes in the stele is modeled as equivalent to a single cylinder with a porous surface 
("leaky pipe”), with the flow inside the stele being axial and approximated as a Poiseuille flow. The axial water volumetric flow rate in the stele, $Q_{W}(z)$, called local water-uptake, is related to the local water pressure in the stele, $p_{s}$, and the local conductivity of the stele, $K_{S}$, by

$$
Q_{W}(z)=-K_{S} \frac{\partial p_{S}}{\partial z},
$$

where $\mathrm{z}$ is the longitudinal direction along the stele. The conductivity of the stele is provided by the xylem vessels and given by

$$
K_{S}=\sum_{i} \frac{\pi n_{i} R_{i}^{4}}{8 \mu},
$$

where $n_{i}, R_{i}$ are the number and the radius of different size class $i$ functional xylem vessels; $\mu$ is the water viscosity. A xylem tube is modeled as a cylinder with a local radius $R_{i}$ which can change along the tube. Gravity is neglected in the mesoscopic single root model. The local water-uptake $Q_{W}(z)$ is related to the water flux density, i.e. flow rate per unit area, entering the stele core from the cortex, $q_{r}$. For the case of a perfect cylindrical porous stele, the flux density $q_{r}$ enters the stele in the radial direction, and it is commonly called the radial flux (density). Conventionally, this radial flux density has been related to the pressure drop across the cortex layer by the membrane-type equation

$$
q_{r}=K_{r}\left(p_{e}-p_{S}\right),
$$

where $p_{e}$ is the water pressure at the root-soil surface, and $K_{r}$ is the so-called radial conductivity of the cortex layer, usually taken as a prescribed constant. The term $p_{e}-p_{s}$ in (5) is the pressure drop across the entire cortex layer at a fixed location $z$. In general, the water pressure on the root-soil surface, $p_{e}$ in (5), depends on water saturation at the root-soil surface. However, as 
discussed above, for the purpose of calculating the internal hydraulic resistance of a root, this root-soil surface pressure can be assumed to be a given constant. Since the radial flux density $q_{r}$ in (5) is proportional to the pressure drop across the entire cortex layer, not to a local pressure gradient, $K_{r}$ is then the overall radial conductivity of the cortex layer. Local water-uptake in the stele and the radial flux density on the stele-cortex interface are related by the mass conservation equation

$$
\frac{\partial Q_{W}}{\partial z}=2 \pi r_{0} q_{r},
$$

where $r_{0}$ is the constant radius of the stele core. When eqns. (5), (6) are combined with eqn. (1), a second-order differential equation for the pressure inside the stele is obtained,

$$
-\frac{\partial}{\partial z}\left(K_{S} \frac{\partial p_{S}}{\partial z}\right)=2 \pi r_{0} K_{r}\left(p_{e}-p_{s}\right) .
$$

This equation is the standard pressure equation for all existing models of water uptake by a single root.

There are several issues with the above approach. On the positive side, the membrane eqn. (5) is global, in the sense that $K_{r}$ is a lumped parameter so that no detailed knowledge of water transport within the cortex layer is required for calculating the radial flux density $q_{r}$ at the stelecortex interface. On the other hand, the radial conductivity of the cortex $K_{r}$ is an overall conductivity, which generally depends not only on the pore size and pore structure of the cortex, but also on the radius of the root $r_{e}$ and the radius of the stele $r_{0}$. This makes it difficult to separate the effect of the cortex physical property from the effect of the root size and stele size on the water-uptake. In addition, eqn. (5) implicitly assumes that the flow in the cortex at any 
given $z$ is planar and purely radial. Additionally, when $K_{r}$ is taken as a constant, eqn. (5) necessarily predicts that the maximum radial flux density on the stele-cortex interface always occurs at the base of the root, since the pressure drop $p_{e}-p_{s}$ is always the highest at the base. At the same time, the minimum radial flux density always occurs at the tip when this theory is applied to a finite-length root, since the pressure drop $p_{e}-p_{s}$ is the lowest at the tip (Fig. 2 of Landsberg \& Fowkes, 1978). Obviously, this kind of radial flux density distribution on the stelecortex interface is incompatible with the water flux density distribution on the root-soil surface for a finite-length root discussed above, with the latter showing an infinitely large flux density near the tip due to the converging flow pattern in the soil.

If, on the other hand, we replace eqn. (5) by Darcy's law in the cortex layer relating the local radial flux to the local radial gradient of the pressure

$$
q_{r}=-\frac{\kappa_{C}}{\mu} \frac{\partial p}{\partial r}
$$

with $\kappa_{C}$ being the permeability of the cortex, then the tip focusing effect in the soil can be reflected in the radial flux density on the stele-cortex interface, $q_{r}$, as the local water pressure gradient $\partial p$ / $\partial r$ in the cortex layer can become very large near the tip. A drawback of using the local eqn. (8) is that one has to find the solution for the water pressure inside the cortex, in addition to the water pressure in the stele. This is, however, a price one must pay in order to maintain the compatibility between the "internal" hydraulics and the "external" hydraulics in a consistent hydraulic resistance model. 
In this paper, we employ a slender-body theory to compute the hydraulic resistance of a single root with end effect in a hydrodynamically consistent manner. The slender-body theory was first used in the analysis of aerodynamic flow around a thin-airfoil and it has been widely used for obtaining approximate solutions for many fluid mechanics problems (Batchelor, 1967). The essence of the slender-body theory is to treat the body as infinitesimally "thin" in a consistent asymptotic expansion. In the present context, the ingredients for such a slender-body treatment have already been laid out in the discussions above. Since the pressure on the root-soil surface $p_{e}$ is maintained at a constant value in the hydraulic resistance calculation, the root is shrunk to a line of finite-length on the z-axis and it is replaced by a line sink (Fig. 4). The solution to the water flow in the saturated soil governed by Darcy's law is unique in this slenderbody limit. This establishes the equivalence between two thin-and-long porous bodies in the slender-body theory: even though the solutions of the flow problems in the soil may not be identical when the sizes of the two bodies are taken into account (in Fig. 4, they are a cylinder and a prolate-spheroid), the two solutions become identical after taking the slender-body limit respectively. Similarly, the internal flows inside the two bodies will also have the same slenderbody limit, as long as the ratio between the root-soil surface and the stele-cortex interface is kept constant when taking the slender-body limit. This allows us to obtain the slender-body limit solution by first solving water-uptake by a finite-size confocal semi prolate-spheroidal root and then taking the required limit (Fig. 4). The advantage of using the prolate-spheroidal root is that the flow solution can be obtained analytically by using a prolate spheroidal coordinate and Legendre functions without the difficulties encountered in dealing with the discontinuous boundary condition when a cylindrical coordinates is used (Chen, 2015). After taking the 
slender-body limit, the resulting formula for the water-uptake and the hydraulic resistance become remarkably simple and easy to use.

It should be pointed out that slender-body theory has been previously applied to water-uptake problems by Miles (1965) and Chu et al. (2008). Both works modeled the root as a cylinder with a variable cross-sectional area and used the slender-body approximation as well as asymptotic matching to solve water flow in a saturated soil. Miles (1965) only considered water flow in the soil which does not provide any result for the root's internal hydraulic resistance. Chu et al. (2008) considered coupled water transport in a saturated soil and the root. In their treatment, however, the entire root is modeled as a single cylinder without the composite structure of a stele and a cortex. In addition, the membrane equation (5) is applied at the soil-root surface, which eliminates the possibility of flux density singularity at the tip. No hydraulic resistance formula was provided by Chu et al. (2008). The present work considers the internal composite coreannular structure of a root; uses Darcy's law (8) instead of the membrane equation (5); and provides an explicit formula for the root's hydraulic resistance. Thus, the results reported here represent a significant progress over these two previous root uptake models based on slenderbody theory.

It must be emphasized that the current work is only limited to the improved calculations of a single root's internal hydraulic resistance. Although the newly obtained hydraulic resistance can be used in the macroscopic scale Richard's equation, which when coupled to the hydraulic equation in a root system, can be used to compute a plant's wateruptake and the water content 
distribution in the soil, no such attempt is made here. Thus issues such as local water depletion and dry-down in the soil cannot be addressed by the present work.

\section{Mathematical formulation}

The hydraulic resistance of a single plant root can be studied by considering water transport within the root, provided this internal flow is hydrodynamically compatible with the external flow in the soil. With the slender-body limit in mind, the root surface is geometrically modeled as one half of a prolate-spheroid with the base of the root located at $z=0$; and once the solution for the prolate-spheroidal root is obtained, the slender-body limit will be taken (Fig. 4). Prolate spheroidal coordinates $(\xi, \eta, \varphi)$ are used, and the radial and angular variables $\tilde{\xi}, \tilde{\eta}$ in a prolate spheroidal coordinates are related to $(\xi, \eta, \varphi)$ by $(\xi, \eta, \varphi)=(\cosh \tilde{\xi}, \cos \tilde{\eta}, \varphi)$ (Smythe, 1989). The Cartesian coordinates $(x, y, z)$ are related to the prolate spheroidal coordinates $(\xi, \eta, \varphi)$ by

$$
x=L \sqrt{\xi^{2}-1} \sqrt{1-\eta^{2}} \cos \varphi, y=L \sqrt{\xi^{2}-1} \sqrt{1-\eta^{2}} \sin \varphi, z=L \xi \eta
$$

where $1 \leq \xi<\infty, 0 \leq \eta \leq 1,0 \leq \varphi \leq 2 \pi$; $L$ is the focal distance. The root surface is described by $\xi=\xi_{e}$. The interface separating the stele from the cortex vessels is modeled similarly as one half of a prolate-spheroid confocal with the root surface, $\xi=\xi_{0}\left(\xi_{0}<\xi_{e}\right) . z_{0}=L \xi_{0}$ is the length of the stele, and $z_{e}=L \xi_{e}$ is the length of the root. At the base of the root, $z=0$, the radius of the stele is $r_{0}=L \sqrt{\xi_{0}^{2}-1}$ and the radius of the root is $r_{e}=L \sqrt{\xi_{e}^{2}-1}$. The stele core and the cortex annulus occupy the regions $1 \leq \xi \leq \xi_{0}$ and $\xi_{0} \leq \xi \leq \xi_{e}$, respectively. The scaling factors for the prolate spheroidal coordinates $(\xi, \eta, \varphi)$ are 


$$
h_{\xi}=L \sqrt{\frac{\xi^{2}-\eta^{2}}{\xi^{2}-1}}, h_{\eta}=L \sqrt{\frac{\xi^{2}-\eta^{2}}{1-\eta^{2}}}, h_{\phi}=L \sqrt{\left(\xi^{2}-1\right)\left(1-\eta^{2}\right)} .
$$

The area elements on the coordinate surfaces are:

$$
\begin{aligned}
& \xi=\text { constant }: d A_{\xi}=h_{\eta} h_{\phi} d \eta d \phi=L^{2} \sqrt{\left(\xi^{2}-\eta^{2}\right)\left(\xi^{2}-1\right)} d \eta d \phi, \\
& \eta=\text { constant }: d A_{\eta}=h_{\xi} h_{\phi} d \xi d \phi=L^{2} \sqrt{\left(\xi^{2}-\eta^{2}\right)\left(1-\eta^{2}\right)} d \xi d \phi .
\end{aligned}
$$

The root surface and the stele-cortex interface are both porous, allowing water to be transported from the soil into the cortex layer, and then from the cortex layer to the stele. Water movements in the cortex and the stele are assumed to obey Darcy's law. For simplicity, the cortex and the stele are assumed to have uniform physical properties, with permeabilities $\kappa_{C}, \kappa_{S}$, respectively. In particular, the permeability of the stele $\kappa_{S}$ is determined by the radius of the xylem tubes. The hydraulic resistance of a root can be studied by assuming the potential (or pressure) on the root surface is maintained at a constant value. Implicitly embedded in the calculation of the hydraulic resistance is the assumption that water demand in the root can always be met by the water supply from the soil (see the Introduction section).

\subsection{The pressure equation in the cortex}

For steady flows, the fluid pressure $p_{C}$ in the cortex satisfies the Laplace equation, which for an axisymmetric flow can be written as

$$
\frac{\partial}{\partial \xi}\left[\left(\xi^{2}-1\right) \frac{\partial p_{C}}{\partial \xi}\right]+\frac{\partial}{\partial \eta}\left[\left(1-\eta^{2}\right) \frac{\partial p_{C}}{\partial \eta}\right]=0
$$


On the root-soil surface $\xi=\xi_{e}$, a constant pressure $p_{e}$ is assumed. Across the stele-cortex interface $\xi=\xi_{0}$, water pressure and the water flux density are continuous.

\subsection{The pressure equation in the stele}

The flow in the stele is predominantly along the z-direction (negative z-direction, or positive $\eta$-direction). Fluid continuity equation is

$$
\frac{\partial}{\partial \xi}\left(L^{2} \sqrt{\left(\xi^{2}-\eta^{2}\right)\left(\xi^{2}-1\right)} \mathrm{v}_{\xi}\right)+\frac{\partial}{\partial \eta}\left(L^{2} \sqrt{\left(\xi^{2}-\eta^{2}\right)\left(1-\eta^{2}\right)} \mathrm{v}_{\eta}\right)=0
$$

where the Darcian velocity is $\mathbf{v}=\mathbf{i}_{\xi} \mathrm{v}_{\xi}+\mathbf{i}_{\eta} \mathrm{v}_{\eta}$. Multiplying the above eqn. by $2 \pi$ and integrating with respect to $\xi$ from $\xi=1$ to $\xi=\xi_{0}$, we obtain

$$
\left.2 \pi L^{2} \sqrt{\left(\xi_{0}^{2}-\eta^{2}\right)\left(\xi_{0}^{2}-1\right)} \mathbf{v}_{\xi}\right|_{\xi=\xi_{0}}+\frac{\partial}{\partial \eta}\left[\int_{1}^{\xi_{0}} 2 \pi L^{2} \sqrt{\left(\xi^{2}-\eta^{2}\right)\left(1-\eta^{2}\right)} \mathbf{v}_{\eta}\right]=0 .
$$

The volumetric flow-rate over a cross-sectional area of the stele core is

$$
Q(\eta)=\int_{1}^{\xi_{0}} 2 \pi L^{2} \sqrt{\left(\xi^{2}-\eta^{2}\right)\left(1-\eta^{2}\right)} \mathrm{v}_{\eta} d \xi
$$

This flow-rate is along the $\eta$-direction. Also

$$
\left.A_{\xi}\right|_{\xi=\xi_{0}, d \eta=1}=\int_{0}^{2 \pi} L^{2} \sqrt{\left(\xi_{0}^{2}-\eta^{2}\right)\left(\xi_{0}^{2}-1\right)} d \phi=2 \pi L^{2} \sqrt{\left(\xi_{0}^{2}-\eta^{2}\right)\left(\xi_{0}^{2}-1\right)}
$$

is the area on the stele-cortex interface per unit increment of $\eta$. Thus, the continuity eqn. (14) can be written as

$$
\frac{\partial Q}{\partial \eta}+\left.\left.A_{\xi}\right|_{\xi=\xi_{0}, d \eta=1} \mathrm{v}_{\xi}\right|_{\xi=\xi_{0}}=0
$$


The second term in the above eqn. is simply the negative of the water influx entering the stele from the cortex through the surface area $\left.A_{\xi}\right|_{\xi=\xi_{0}, d \eta=1}$.

The surface water influx into the stele can be simplified by using Darcy's law for water movement in the cortex

$$
\mathbf{v}_{C}=-\frac{\kappa_{C}}{\mu} \nabla p_{C} .
$$

This gives

$$
\left.\mathrm{v}_{\xi}\right|_{\xi=\xi_{0}}=-\left.\frac{\kappa_{C}}{\mu} \frac{1}{L} \sqrt{\frac{\xi_{0}^{2}-1}{\xi_{0}^{2}-\eta^{2}}} \frac{\partial p_{C}}{\partial \xi}\right|_{\xi=\xi_{0}} .
$$

Thus,

$$
\left.\left.A_{\xi}\right|_{\xi=\xi_{0}, d \eta=1} \mathrm{v}_{\xi}\right|_{\xi=\xi_{0}}=-\left.\frac{2 \pi \kappa_{C}}{\mu} L\left(\xi_{0}^{2}-1\right) \frac{\partial p_{C}}{\partial \xi}\right|_{\xi=\xi_{0}} .
$$

In the stele,

$$
\begin{aligned}
& \mathbf{v}_{S}=-\frac{\kappa_{S}}{\mu} \nabla p_{S}, \\
& \mathrm{v}_{\eta}=-\frac{\kappa_{S}}{\mu} \frac{1}{L} \frac{\sqrt{1-\eta^{2}}}{\sqrt{\xi^{2}-\eta^{2}}} \frac{\partial p_{S}}{\partial \eta} .
\end{aligned}
$$

Thus, from (15), the volumetric flow rate in the stele becomes

$$
Q(\eta)=-\frac{2 \pi \kappa_{S}}{\mu} L\left(\xi_{0}-1\right)\left(1-\eta^{2}\right) \frac{\partial \bar{p}}{\partial \eta},
$$

where the average pressure $\bar{p}$ is defined as 


$$
\bar{p}=\frac{\int_{1}^{\xi_{0}} p_{s} d \xi}{\xi_{0}-1} .
$$

The continuity eqn. (17) is then reduced to an eqn. for the average pressure $\bar{p}$ in the stele as

$$
\frac{d}{d \eta}\left[\left(1-\eta^{2}\right) \frac{d \bar{p}}{d \eta}\right]+\left.\frac{\xi_{0}+1}{\lambda} \frac{\partial p_{C}}{\partial \xi}\right|_{\xi=\xi_{0}}=0
$$

where

$$
\lambda=\frac{\kappa_{S}}{\kappa_{C}}
$$

is the ratio between the stele permeability and the cortex permeability, which plays a key role in the root water uptake.

The pressure in the cortex $p_{C}(\xi, \eta)$ and the average pressure inside the stele $\bar{p}(\eta)$ can be obtained by solving eqns. (12) and (25) with the conditions of continuity in the pressure and the flux density at the stele-cortex interface; as well as the following boundary conditions:

$$
\begin{array}{ll}
\xi=\xi_{e}: & p=p_{e}(\text { root surface pressure }) ; \\
\eta=1: & \text { symmetry about z-axis } \\
& \frac{d p_{C}}{d \eta}=0 ; \frac{d \bar{p}}{d \eta}=0 ; \\
\eta=0: & \left.\frac{d p_{C}}{d \eta}=0 \text { (no-flow acrossing } \mathrm{x}-\text { axis }\right) ; \\
& \left.\bar{p}=p_{w} \text { (given stele pressure at the base of the root }\right) .
\end{array}
$$

The method of separation of variables is used for solving the above boundary-value problem. The pressure distribution in the cortex is found to be given by

$$
p_{C}(\xi, \eta)=\sum_{m=0}^{\infty}\left[A_{2 m} P_{2 m}(\xi)+B_{2 m} Q_{2 m}(\xi)\right] P_{2 m}(\eta), \xi_{0} \leq \xi<\xi_{e}, 0 \leq \eta \leq 1
$$


where $P_{2 m}$ and $Q_{2 m}$ are the zeroth-order associated Legendre function of the first and the second kind, respectively. The average pressure inside the stele $\bar{p}(\eta)$ is given by $\bar{p}(\eta)$
$=p_{w}+\frac{B_{0} Q_{0}^{\prime}\left(\xi_{0}\right)}{\lambda}\left(\xi_{0}+1\right) \ln (1+\eta)+\frac{\xi_{0}+1}{\lambda} \sum_{m=1}^{\infty} \frac{A_{2 m} P_{2 m}^{\prime}\left(\xi_{0}\right)+B_{2 m} Q_{2 m}^{\prime}\left(\xi_{0}\right)}{2 m(2 m+1)}\left[P_{2 m}(\eta)-P_{2 m}(0)\right], 0 \leq \eta \leq 1$,

where primes stand for derivatives. The coefficients $\left\{A_{2 m}, B_{2 m}\right\}$ are given in the Appendix.

\section{The slender-body limit}

The leading order solution in the slender-body theory can be obtained from the general solution given in Section 2 by taking the $\operatorname{limit}\left(\xi_{0}, \xi_{e}\right) \rightarrow(1,1)$ while maintaining the ratio $\xi_{e} / \xi_{0}$ $(>1)$, and $\lambda\left(\xi_{0}^{2}-1\right)=$ constant. To this end, we define a new dimensionless parameter $C_{s D}$ :

$$
C_{s D}=\lambda\left(\xi_{0}^{2}-1\right)=\frac{\kappa_{S} r_{0}^{2}}{\kappa_{C} L^{2}}
$$

The role the parameter $C_{s D}$ plays in root-water uptake is similar to the role the dimensionless fracture conductivity $C_{f D}$ plays in fluid production from a hydraulic fracture (Cinco-Ley et al., 1978). $r_{0}^{2}$ characterizes the cross-sectional area over which water flows through in the stele; and $C_{s D}$ is a dimensionless conductivity of the stele which measures the hydraulic conductivity of the stele relative to that of the cortex. For a thin root to be effective in water-uptake, $C_{s D}$ needs to be at least an order one quantity. There is a significant difference between a fracture and a root, however: when a fracture becomes very thin, the fluid-producing area of a fracture remains constant due to its two-dimensionality; while the fluid-producing area of a root's surface shrinks to zero. Root water-uptake goes to zero when $C_{s D} \rightarrow 0$, as will be shown in later sections. 
From eqn. (19),

$$
\left.\mathrm{v}_{\xi}\right|_{\xi=\xi_{0}}=-\left.\frac{\kappa_{C}}{\mu} \frac{1}{L} \sqrt{\frac{\xi_{0}^{2}-1}{\xi_{0}^{2}-\eta^{2}}} \frac{\partial p_{C}}{\partial \xi}\right|_{\xi=\xi_{0}} .
$$

In the limit of slender-body theory, the water flux density of the fluid entering the stele through the stele-cortex interface called radial flux (density) becomes

$$
q_{s}(\eta)=-\left.\mathrm{v}_{\xi}\right|_{\xi=\xi_{0}}=\frac{\kappa_{C}}{\mu} \frac{1}{L} \sqrt{\frac{\xi_{0}^{2}-1}{1-\eta^{2}}}\left\{B_{0} Q_{0}^{\prime}\left(\xi_{0}\right)+\sum_{m=1}^{\infty}\left[A_{2 m} P_{2 m}^{\prime}\left(\xi_{0}\right)+B_{2 m} Q_{2 m}^{\prime}\left(\xi_{0}\right)\right] P_{2 m}(\eta)\right\} .
$$

In the slender-body limit, $\eta=z / L$. Since $P_{2 m}(1)$ is bounded, eqn. (32) shows that the water flux density at the stele-cortex interface has an inverse-square-root singularity in the distance from the root tip $\eta=1$. This tip singularity is consistent with the same type of tip singularity for the water flux density entering the root from the root-soil surface (Chen, 2015).

Another key element in the slender-body approximation is to replace the surface area element on the stele-cortex interface by the area corresponding to a perfectly cylindrical interface. Thus,

$$
\left.A_{\xi}\right|_{\xi=\xi_{0}, d \eta=1} \rightarrow 2 \pi r_{0} L=2 \pi L^{2} \sqrt{\xi_{0}^{2}-1}
$$

The continuity eqn. (17) now becomes

$$
\frac{d Q}{d \eta}=2 \pi L \frac{\kappa_{C}}{\mu} \frac{\xi_{0}^{2}-1}{\sqrt{1-\eta^{2}}}\left\{B_{0} Q_{0}^{\prime}\left(\xi_{0}\right)+\sum_{m=1}^{\infty}\left[A_{2 m} P_{2 m}^{\prime}\left(\xi_{0}\right)+B_{2 m} Q_{2 m}^{\prime}\left(\xi_{0}\right)\right] P_{2 m}(\eta)\right\}
$$

Integrating (34), taking into account that $Q(1)=0$ (at the tip), we then have

$$
Q(\eta)=\frac{2 \pi L \kappa_{C}}{\mu}\left(\xi_{0}^{2}-1\right)\left\{B_{0} Q_{0}^{\prime}\left(\xi_{0}\right)\left(\sin ^{-1} \eta-\frac{\pi}{2}\right)+\sum_{m=1}^{\infty}\left[A_{2 m} P_{2 m}^{\prime}\left(\xi_{0}\right)+B_{2 m} Q_{2 m}^{\prime}\left(\xi_{0}\right)\right] \int_{1}^{\eta} \frac{P_{2 m}(\sigma)}{\sqrt{1-\sigma^{2}}} d \sigma\right\}
$$


Thus $Q(\eta)$ is the volumetric flow rate along the z-direction in the stele. However, water in the stele moves towards the base of the root, $\eta=0(z=0)$. Thus, the local water-uptake $Q_{W}(\eta)$ is the negative of $Q(\eta)$, and it is given by

$$
Q_{W}(\eta)=\frac{2 \pi L \kappa_{C}}{\mu}\left(\xi_{0}^{2}-1\right)\left\{B_{0} Q_{0}^{\prime}\left(\xi_{0}\right)\left(\frac{\pi}{2}-\sin ^{-1} \eta\right)+\sum_{m=1}^{\infty}\left[A_{2 m} P_{2 m}^{\prime}\left(\xi_{0}\right)+B_{2 m} Q_{2 m}^{\prime}\left(\xi_{0}\right)\right] \int_{\eta}^{1} \frac{P_{2 m}(\sigma)}{\sqrt{1-\sigma^{2}}} d \sigma\right\} .
$$

The total water-uptake rate at the base $\eta=0$ is then

$$
Q_{W}(0)=\frac{2 \pi L \kappa_{C}}{\mu}\left(\xi_{0}^{2}-1\right)\left\{\frac{\pi}{2} B_{0} Q_{0}^{\prime}\left(\xi_{0}\right)+\sum_{m=1}^{\infty}\left[A_{2 m} P_{2 m}^{\prime}\left(\xi_{0}\right)+B_{2 m} Q_{2 m}^{\prime}\left(\xi_{0}\right)\right] \int_{0}^{1} \frac{P_{2 m}(\sigma)}{\sqrt{1-\sigma^{2}}} d \sigma\right\} .
$$

The total hydraulic resistance of the root is given by

$$
R_{T}=\frac{\pi r_{0}^{2} \Delta p}{Q_{W}(0)}
$$

\section{The flux density distribution on the stele-cortex interface}

In the slender-body limit, the variable $\eta$ becomes the dimensionless $\mathrm{z}$

$$
\eta=\frac{Z}{L}=\hat{z}
$$

We introduce a dimensionless flux density on the stele-cortex interface, $\hat{q}_{s}(\eta)$ :

$$
\hat{q}_{s}(\eta)=\frac{q_{s}(\eta) \mu L}{\kappa_{C} \Delta p}=\frac{1}{h\left(C_{s D}, \xi_{0}, \xi_{e}\right)}\left\{\frac{1}{\sqrt{\xi_{0}^{2}-1}} \frac{1}{\sqrt{1-\eta^{2}}}-\frac{\sqrt{\xi_{0}^{2}-1}}{\sqrt{1-\eta^{2}}} \sum_{m=1}^{\infty}\left[a_{2 m} P_{2 m}^{\prime}\left(\xi_{0}\right)+b_{2 m} Q_{2 m}^{\prime}\left(\xi_{0}\right)\right] P_{2 m}(\eta)\right\}
$$

where

$$
h\left(C_{s D}, \xi_{0}, \xi_{e}\right)=Q_{0}\left(\xi_{0}\right)-Q_{0}\left(\xi_{e}\right)+\left(\xi_{0}+1\right) \frac{2 \ln 2-1-\mathrm{g}\left(C_{s D}, \xi_{0}, \xi_{e}\right)}{C_{s D}},
$$




$$
\begin{aligned}
& g\left(C_{s D}, \xi_{0}, \xi_{e}\right)=\sum_{m=1}^{\infty} \frac{(4 m+1)\left(\xi_{0}+1\right) I_{2 m} P_{2 m}(0)}{2 m(2 m+1) \frac{C_{s D}}{\xi_{0}^{2}-1} \frac{Q_{2 m}\left(\xi_{0}\right) P_{2 m}\left(\xi_{e}\right)-P_{2 m}\left(\xi_{0}\right) Q_{2 m}\left(\xi_{e}\right)}{Q_{2 m}^{\prime}\left(\xi_{0}\right) P_{2 m}\left(\xi_{e}\right)-P_{2 m}^{\prime}\left(\xi_{0}\right) Q_{2 m}\left(\xi_{e}\right)}-\left(\xi_{0}+1\right)}, \\
& b_{2 m}=\frac{2 m(2 m+1)(4 m+1)\left(\xi_{0}+1\right) I_{2 m} Q_{0}^{\prime}\left(\xi_{0}\right) P_{2 m}\left(\xi_{e}\right)}{2 m(2 m+1) \frac{C_{s D}}{\xi_{0}^{2}-1}\left[Q_{2 m}\left(\xi_{0}\right) P_{2 m}\left(\xi_{e}\right)-P_{2 m}\left(\xi_{0}\right) Q_{2 m}\left(\xi_{e}\right)\right]-\left(\xi_{0}+1\right)\left[Q_{2 m}^{\prime}\left(\xi_{0}\right) P_{2 m}\left(\xi_{e}\right)-P_{2 m}^{\prime}\left(\xi_{0}\right) Q_{2 m}\left(\xi_{e}\right)\right]}, \\
& a_{2 m}=-b_{2 m} \frac{Q_{2 m}\left(\xi_{e}\right)}{P_{2 m}\left(\xi_{e}\right)}, m \geq 1 .
\end{aligned}
$$

One observation is that when the dimensionless stele conductivity $C_{s D} \rightarrow 0$, $h\left(C_{s D}, \xi_{0}, \xi_{e}\right) \rightarrow \infty$; As a result, the flux density $\hat{q}_{s}(\eta)$ becomes zero everywhere. Physically, in this limit, the stele has an infinitely large (axial) resistance, and there will be no water flowing through the stele. Clearly, as eqn. (40) shows, as long as $C_{s D}>0$, the flux density $\hat{q}_{s}(\eta)$ also possesses an inverse-square-root singularity at the tip.

The dimensionless flux density distribution on the stele-cortex surface $\hat{q}_{s}(\hat{z})$ is plotted in Fig. 5 for a slender root with $r_{0} / L=0.01, r_{e} / L=0.05$ and dimensionless stele conductivities $C_{s D}=0.6,0.8,1,2,5,20,50,100$. The inverse-square-root flux density singularity near the root tip manifests itself prominently in these plots. As shown in Fig. 5, there are two types of flux density curves: for large values of $C_{s D}\left(C_{s D}=5,20,50,100\right)$, the flux density increases monotonically in the direction towards the tip; while for smaller $C_{s D}\left(C_{s D}=0.6,0.8,1,2\right)$, the flux density first decreases, then followed by a rapid increase as the tip is approached. For both types of flux density curves, the flux density becomes unbounded at the tip due to the inverse-square-root singularity. Since the area under the flux density curve for any segment measured from the tip is the water volumetric flow rate entering that segment of the stele from the cortex, per unit length 
water-uptake is always the largest near the tip for large values of $C_{s D}$. It is also observed that the flux densities for $C_{s D}=50$ and $C_{s D}=100$ are almost identical, both are nearly the same as that for the maximum flux density, corresponding to infinitely large stele conductivity $C_{s D}=\infty$.

The flux density distribution on the stele-cortex interface $\hat{q}_{s}(\hat{z})$ for a similar root with a thinner cortex layer, $r_{0} / L=0.01, r_{e} / L=0.03$ are plotted in Fig. 6, with the same dimensionless stele conductivities $C_{S D}=0.6,0.8,1,2,5,20,50,100$. These flux density curves have the same features as those for $r_{0} / L=0.01, r_{e} / L=0.05$ shown in Fig. 5, with two distinct types of curves. The only difference is that the values of the dimensionless flux densities are markedly higher for the case of a thinner cortex layer shown in Fig. 6. This is due to a lower overall "radial" resistance from the thinner cortex layer, allowing a higher water flow rate per unit area across the cortex layer for a given pressure drop across the cortex layer. Another case for an even thinner cortex layer with $r_{0} / L=0.01, r_{e} / L=0.02$ is shown in Fig. 7. The flux density curves for small stele conductivities $C_{s D}=0.6,0.8,1$ show a much steeper dip towards the tip before rising rapidly to very large values close to the tip due to the tip singularity.

The change in the shape of the flux density $\hat{q}_{s}(\eta)$ curve with the dimensionless stele conductivity, or axial conductivity, $C_{\mathrm{sD}}$, reflects the competition between two mechanisms for root water-uptake: the lowest pressure in the root is at the base, which tends to draw most water in the soil to the region near the base; and the largest water pressure gradient in the soil is near the root tip, which converges water to the tip region. Which mechanism prevails is determined 
by the dimensionless stele conductivity $C_{s D}$. The underlying physics has been examined in detail by Chen (2015). In the present context, the same mechanisms work in unison in both the soil and inside the root's own composite structure of a stele core and a cortex annulus. As we have discussed extensively before, the flux density on the stele-cortex interface $\hat{q}_{s}(\eta)$ also reflects how water enters the root from the soil. While we do not attempt to find the water flow solution under the general unsaturated soil condition here, the solution for the water pressure in the soil with a constant pressure on the root-soil surface in an unbounded saturated soil can be easily found to be

$$
p_{\text {Soil }}(\xi, \eta)=p_{\infty}-\left(p_{\infty}-p_{e}\right) \frac{\ln (\xi+1)-\ln (\xi-1)}{\ln \left(\xi_{e}+1\right)-\ln \left(\xi_{e}-1\right)}, \xi_{e} \leq \xi<\infty,
$$

where $p_{\infty}$ is the far field water pressure in the soil. The water flux density at the root-soil surface in the slender-body limit is given by

$$
q_{\text {root-soil }}=\frac{\kappa_{\text {soil }}}{\mu} \frac{2}{L} \frac{p_{\infty}-p_{e}}{\ln \left(\xi_{e}+1\right)-\ln \left(\xi_{e}-1\right)} \frac{1}{\sqrt{\xi_{e}^{2}-1}} \frac{1}{\sqrt{1-\eta^{2}}},
$$

which clearly shows an inverse-square-root singularity at the root tip. The pattern of the water flow in the soil shown Fig. 2 is created based on the solution (42). Water flow focusing onto the tip region is clearly demonstrated in Fig. 2. This flow characteristic in the soil also causes the flux density on the stele-cortex interface $\hat{q}_{s}(\eta)$ to behavior similarly near the tip as that of the flux density on the root-soil surface, with a flux density singularity at the tip in the slender-body limit, as shown in the solution (40) and Fig. 5-7. This integrable "radial” flux density singularity reflects the three-dimensional nature of the external water flow in the soil near the tip of a root; and it is completely absent from the traditional purely radial flow theory of Landsberg \& Fowkes (1978). 
The water-uptake competition landscape is exemplified by the flux density curves for representative low and high values of the axial conductivity, $C_{s D}=0.2,20$, in Fig. 8 for $r_{0} / L=0.01, r_{e} / L=0.03$, with the intermediate values $C_{s D}=1,5$ serving as reference. The dominance of the basal region in water-uptake is evident in low axial conductivity flux density curve $C_{s D}=0.2$ : the value of the flux density $\hat{q}_{s}(\hat{z})$ for $\hat{z}>0.6$ drops to about $25 \%$ of the flux density value at the base. The high flux density zone around the tip is narrowed to a very narrow window, with a small area underneath that portion of the curve. This is in sharp contrast to flux density curve for high axial conductivity $C_{s D}=20$, where the tip region manifests its dominance with a large area underneath the portion of the flux density curve near the tip. Shifting of "water production” from the basal region to the tip region is clearly demonstrated as $C_{s D}$ is increased from 0.2 to 20. It is worth noting that Zwieniecki et al. (2003) employed the analogy of using a porous straw to drink a martini out of a cup to describe this competition between the two water production mechanisms without the benefit of having the flux density curves. Tip effect was absent in their discussions and their description was only qualitative.

\section{Local water uptake profiles in the stele}

Define a local dimensionless water-uptake as

$$
\hat{Q}_{W}(\hat{z})=\frac{Q_{W}(\hat{z})}{2 \pi L \sqrt{\xi_{0}^{2}-1}} \frac{\mu}{\kappa_{C} \Delta p} .
$$

Then the continuity eqn. (17) gives

$$
\frac{\partial \hat{Q}_{w}}{\partial \hat{z}}=-\hat{q}_{s}(\hat{z}),
$$


which can be integrated to give

$$
\hat{Q}_{w}(\hat{z})=\frac{1}{h\left(C_{s D}, \xi_{0}, \xi_{e}\right)}\left\{\frac{1}{\sqrt{\xi_{0}^{2}-1}}\left(\frac{\pi}{2}-\sin ^{-1} \hat{z}\right)-\sqrt{\xi_{0}^{2}-1} \sum_{m=1}^{\infty}\left[a_{2 m} P_{2 m}^{\prime}\left(\xi_{0}\right)+b_{2 m} Q_{2 m}^{\prime}\left(\xi_{0}\right)\right] \int_{\hat{z}}^{1} \frac{P_{2 m}(\sigma)}{\sqrt{1-\sigma^{2}}} d \sigma\right\} .
$$

Since the water-uptake is a maximum at the base of the root (i.e. total water-uptake by the stele), we plot the normalized water-uptake profile $\hat{Q}_{w}(\hat{z}) / \hat{Q}_{w}(0)$ for $r_{0} / L=0.01 ; r_{e} / L=0.03$ $C_{s D}=0.6,0.8,1,2,5,20,50,100$ in Fig. 9. Water-uptake increases with the axial conductivity $C_{s D}$; and the uptake profile for $C_{s D}=20$ already approaches that for the upper limit of $C_{s D} \rightarrow \infty$. Another prominent feature of the uptake profile is that the increase in the uptake from zero at the root tip is very rapid. In fact, the inverse-square-root singularity in the flux density $\hat{q}_{s}(\hat{z})$ indicates that the uptake $\hat{Q}_{w}(\hat{z})$ increases as the square-root of the distance to the tip. The uptake, however, slows its rate of increase to a nearly linear rate in the distance from the tip as the root base is approached. It is observed from Fig. 9 that away from the rapid rise in near tip region, the water-uptake profiles for large axial conductivity $C_{s D} \geq 2$ are concave while those for smaller axial conductivity $C_{s D} \leq 1$ are convex. Near the base of the root, $\hat{z} \rightarrow 0$, all water-uptake curves become linear, but with a different slope,

$$
\hat{Q}_{w}(\hat{z})=\frac{1}{h\left(C_{s D}, \xi_{0}, \xi_{e}\right)} \frac{1}{\sqrt{\xi_{0}^{2}-1}}\left(\frac{\pi}{2}-\hat{z}\right)
$$

In particular, in the large axial conductivity limit $C_{s D} \rightarrow \infty$, the straight-line near the base of dimensionless water-uptake becomes 


$$
\hat{Q}_{w}(\hat{z})=\frac{2}{\ln \left(\xi_{e}-1\right)-\ln \left(\xi_{0}-1\right)} \frac{1}{\sqrt{\xi_{0}^{2}-1}}\left(\frac{\pi}{2}-\hat{z}\right)
$$

We have plotted this limiting straight-line in Fig. 10, together with the normalized local wateruptake for $r_{0} / L=0.01 ; r_{e} / L=0.03 C_{s D}=0.2,0.6,1,5,20$. It is obvious from Fig. 10 that wateruptake departs significantly from the linear rate of increase along the root. There is always a rapid nonlinear rise in water-uptake near the tip, caused by the flux density singularity.

It must be pointed out that since the pressure inside the cortex $p_{C}(\xi, \eta)$ changes with $\eta$ (see (28)), there will be an axial movement of water within the cortex as well. This means that not all water that has entered the root from the soil will enter the stele; and the water-uptake in the stele will be generally less than the amount of water that entered the root from the soil. However, this difference is small when the dimensionless axial conductivity $C_{s D}$ is large, as the axial variation of $p_{C}(\xi, \eta)$ becomes negligible for large $C_{s D}\left(a_{2 m}, b_{2 m}, m \geq 1\right.$ go to zero as $C_{s D}$ becomes large).

Zwieniecki et al. (2003) measured the local water-uptake along a root by using successive distal and proximal cuts. They normalized the local uptake by the uptake at the base and the distance to the tip by length of the root, and presented their data in Fig. 5 in their paper. These measurements show significant scattering, even with a few negative local water uptake data points. Zwieniecki et al. also performed numerical calculations for water-uptake with a cylinder model for given ratios between the longitudinal (axial) conductivity and the radial (global) conductivity, and plotted these numerical results together with the measured values. They concluded that the longitudinal conductivity to the radial conductivity ratio of 0.025 provides the best fit to the measured data. However, it is observed from their plots that the measured local water-uptake values deviate significantly from this computed local uptake profile throughout the 
entire length of the root. In fact, it is quite evident that it is not possible to fit these measured data points into a single curve. Since the measured local uptake values were obtained from measurement made on successive cut of the original root, the inability to fit the data into a single curve indicates that local water-uptake depends on the length of the root after the cut, not just the original length of the root. This point of view is supported by the current slender-body theory as illustrated in Fig. 10: the local water-uptake profile depends on the dimensionless parameter $C_{s D}=\kappa_{S} r_{0}^{2} /\left(\kappa_{C} L^{2}\right)$, which will be increased when the original root is cut short ( $L$ becomes smaller). Thus it is impossible to fit the data of Zwieniecki et al. (2003) into a single uptake curve, as each measured data point corresponds to a different value of $C_{s D}$. The ratio between the longitudinal (axial) conductivity and the radial conductivity used in the calculations of Zwieniecki et al. (2003) is the same for the same root, from the original entire root to all the successive cuts; this ratio between the two conductivities cannot capture the dependence of the local uptake on the length of the newly cut root.

\section{The overall water-uptake rate in the stele and the hydraulic resistance of a root}

The overall water-uptake of a root is the water-uptake at the base of the stele:

$$
\hat{Q}_{O}=\hat{Q}_{w}(0)=\frac{1}{h\left(C_{s D}, \xi_{0}, \xi_{e}\right)}\left\{\frac{\pi}{2} \frac{1}{\sqrt{\xi_{0}^{2}-1}}-\sqrt{\xi_{0}^{2}-1} \sum_{m=1}^{\infty}\left[a_{2 m} P_{2 m}^{\prime}\left(\xi_{0}\right)+b_{2 m} Q_{2 m}^{\prime}\left(\xi_{0}\right)\right] \int_{0}^{1} \frac{P_{2 m}(\sigma)}{\sqrt{1-\sigma^{2}}} d \sigma\right\} .
$$

In dimensional form, the overall water-uptake is given by (from (44), (45)):

$$
Q_{O}=\frac{\kappa_{C} \Delta p}{\mu} \frac{2 \pi L}{h\left(C_{s D}, \xi_{0}, \xi_{e}\right)}\left\{\frac{\pi}{2}-\left(\xi_{0}^{2}-1\right) \sum_{m=1}^{\infty}\left[a_{2 m} P_{2 m}^{\prime}\left(\xi_{0}\right)+b_{2 m} Q_{2 m}^{\prime}\left(\xi_{0}\right)\right] \int_{0}^{1} \frac{P_{2 m}(\sigma)}{\sqrt{1-\sigma^{2}}} d \sigma\right\} .
$$

The overall hydraulic resistance of a root $R_{\text {Total }}$ is explicitly given by 


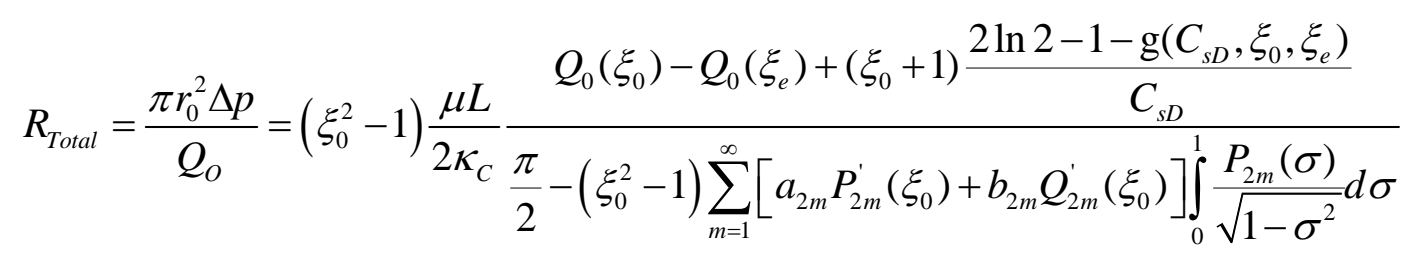

The dimensionless total water-uptake $\hat{Q}_{O}$ is plotted against the dimensionless axial conductivity $C_{s D}$ for $r_{0} / L=0.01 ; r_{e} / L=0.02,0.03,0.05$ in Fig. 11 . With everything else being equal, a thinner cortex layer (smaller value of $r_{e} / L$ ) provides a higher dimensionless $\hat{Q}_{O}$. This is caused by the reduction in the radial resistance for a thinner cortex annulus. Also evident from Fig. 11 is that as the dimensionless axial conductivity $C_{s D}$ increases from small values, $\hat{Q}_{O}$ increase rapidly. $\hat{Q}_{O}$, however, plateaus as $C_{s D}$ is increased beyond certain values; it essentially approaches the limiting value for infinite dimensionless axial conductivity.

Further insights can be gained by inspecting the overall water-uptake rate in the limit of large dimensionless axial conductivity $C_{s D}$. From (41), for large $C_{s D}$, both $a_{2 m}, b_{2 m}$ become very small $\left(O\left(1 / C_{c D}\right)\right)$, and the infinite sum in the bracket on the right-hand-side of (49) becomes a higherorder term. Furthermore, (41) also shows that $\mathrm{g}\left(C_{\mathrm{SD}}, \xi_{0}, \xi_{e}\right)$ becomes a high-order term for large $C_{s D}$. Thus, for large $C_{s D}$,

$$
\hat{Q}_{O} \approx \frac{\pi}{2} \frac{1}{\sqrt{\xi_{0}^{2}-1}} \frac{1}{\frac{1}{2}\left(\ln \frac{\xi_{0}+1}{\xi_{0}-1}-\ln \frac{\xi_{e}+1}{\xi_{e}-1}\right)+\left(\xi_{0}+1\right) \frac{2 \ln 2-1}{C_{S D}}},
$$


which in the slender-body limit simplifies to

$$
\hat{Q}_{O} \approx \frac{\pi}{\sqrt{\xi_{0}^{2}-1}} \frac{1}{\ln \frac{\xi_{e}-1}{\xi_{0}-1}+4 \frac{2 \ln 2-1}{C_{s D}}} .
$$

The corresponding dimensional overall water-uptake rate and total hydraulic resistance become

$$
\begin{aligned}
& Q_{O} \approx \frac{\kappa_{C} \Delta p}{\mu} \frac{\pi^{2} L}{\ln \frac{r_{e}}{r_{0}}+2 \frac{2 \ln 2-1}{C_{S D}},} \\
& R_{\text {Total }} \approx \frac{\mu L}{\pi \kappa_{C}}\left(\xi_{0}^{2}-1\right)\left[\ln \frac{r_{e}}{r_{0}}+\frac{0.7726}{C_{s D}}\right] .
\end{aligned}
$$

Eqns. (54), (55) provide the leading-order expressions for the total water-uptake and the total hydraulic resistance, respectively, for large but finite values of axial conductivity $C_{\mathrm{sD}}$ in the slender-body limit. For this case, the total hydraulic resistance is the sum of a radial resistance and an axial resistance,

$$
R_{\text {Total }}=R_{\text {Radial }}+R_{\text {Axial }},
$$

with

$$
\begin{aligned}
& R_{\text {Radial }}=\frac{\mu L}{\pi \kappa_{C}}\left(\xi_{0}^{2}-1\right) \ln \frac{r_{e}}{r_{0}}=\frac{\mu r_{0}^{2}}{\pi \kappa_{C} L} \ln \frac{r_{e}}{r_{0}}, \\
& R_{\text {Axial }}=\frac{0.7726}{C_{s D}} \frac{\mu L}{\pi \kappa_{C}}\left(\xi_{0}^{2}-1\right)=0.7726 \frac{\mu L}{\pi \kappa_{S}} .
\end{aligned}
$$

In particular, for infinitely large axial conductivity, $C_{s D} \rightarrow \infty$, the axial resistance term in the denominator of eqn. (54) is negligible, and we have

$$
Q_{O}=\frac{\kappa_{C} \Delta p}{\mu} \frac{\pi^{2} L}{\ln \left(r_{e} / r_{0}\right)} .
$$

On the other hand, the infinite-cylinder model of Landsberg \& Fowkes (1978) gives 


$$
Q_{L F}=\frac{\kappa_{C} \Delta p}{\mu} \frac{2 \pi L}{\ln \left(r_{e} / r_{0}\right)} .
$$

Thus, our slender-body model predicts 57\% more overall water-uptake than the Landsberg \& Fowkes model:

$$
\frac{Q_{O}}{Q_{L F}}=\frac{\pi}{2}=1.57 .
$$

This increase in the water-uptake is due to the three-dimensional focusing of water flow in the soil to the tip. In terms of the total hydraulic resistance, we have

$$
\frac{R_{\text {Total }}}{R_{L F}}=\frac{2}{\pi}=0.64 .
$$

Thus, for this particular case, the hydraulic resistance of the slender-body model is $36 \%$ lower than that predicted by Landsberg \& Fowkes (1978).

The general total hydraulic resistance formula given by eqn. (51) is complicated; and it cannot be decomposed into a simple sum of a radial and an axial resistance because of the non-

trivial denominator. After all, an analogy to an electrical network requires that all the flows involved are rectilinear. Concepts such as "resistors in serial" etc. are not applicable to nonrectilinear flows, such as the case for a finite stele axial conductivity.

\section{Conclusions}

This work presents a slender-body theory for the calculation of the internal hydraulic resistance of a single root. The work provides an in-depth discussion on the procedure and the assumptions involved in calculating the internal hydraulic resistance; as well as the physical and the mathematical aspects of the external three-dimensional water flow around the tip of a root 
and how this converging flow pattern influences internal hydraulic resistance of a single root. The analysis shows that compatibility between the external flow and the internal flow causes the flux density on the stele-cortex interface to possess large values in the near tip region. In the slender-body limit, analytical solutions for the flux density distribution on the stele-cortex interface, local water-uptake profile inside the stele core, and the overall water-uptake at the base of the stele are obtained using a composite confocal ellipsoid root model. It is shown that a key parameter controlling the hydraulic resistance is the dimensionless axial conductivity in the stele, $C_{s D}$, which depends on the permeabilities in the stele and the cortex as well as the stele radial and axial dimensions. Three-dimensional tip effect reduces the root's hydraulic resistance by as much as $36 \%$ when compared to the radial flow theory of Landsberg \& Fowkes (1978). A general and explicit analytical expression for the total hydraulic resistance of a root is given; and the total hydraulic resistance cannot be decomposed into the sum of a radial resistance and an axial resistance. Only for large axial conductivities the total hydraulic resistance can be written as the sum of a radial resistance and an axial resistance, for which a simple to use approximate formula is provided. The result from the present study can be readily adopted in the macroscopic scale models used to study water-uptake by a plant's root system.

Acknowledgement This work is supported by the National Science Foundation (CBET 0932968) and the Donors of the Petroleum Research Fund, administered by the American Chemical Society (52177-ND9).

Appendix $A_{2 m}, B_{2 m}$ and $H\left(\lambda, \xi_{0}, \xi_{e}\right)$

$\Delta p=p_{e}-p_{w}$, 


$$
B_{0}=-\frac{\xi_{0}^{2}-1}{H\left(\lambda, \xi_{0}, \xi_{e}\right)} \Delta p
$$

$m \geq 1$ :

$B_{2 m}=\frac{2 m(2 m+1)(4 m+1)\left(\xi_{0}+1\right) I_{2 m} Q_{0}^{\prime}\left(\xi_{0}\right) P_{2 m}\left(\xi_{e}\right)}{2 m(2 m+1) \lambda\left[Q_{2 m}\left(\xi_{0}\right) P_{2 m}\left(\xi_{e}\right)-P_{2 m}\left(\xi_{0}\right) Q_{2 m}\left(\xi_{e}\right)\right]-\left(\xi_{0}+1\right)\left[Q_{2 m}^{\prime}\left(\xi_{0}\right) P_{2 m}\left(\xi_{e}\right)-P_{2 m}^{\prime}\left(\xi_{0}\right) Q_{2 m}\left(\xi_{e}\right)\right]} B_{0}$ ;

$$
A_{0}=p_{e}-B_{0} Q_{0}\left(\xi_{e}\right)
$$

$$
A_{2 m}=-B_{2 m} \frac{Q_{2 m}\left(\xi_{e}\right)}{P_{2 m}\left(\xi_{e}\right)}, m \geq 1
$$$$
H\left(\lambda, \xi_{0}, \xi_{e}\right)=\left(\xi_{0}^{2}-1\right)\left(Q_{0}\left(\xi_{0}\right)-Q_{0}\left(\xi_{e}\right)\right)+\left(\xi_{0}+1\right) \frac{2 \ln 2-1-\mathrm{g}\left(\lambda, \xi_{0}, \xi_{e}\right)}{\lambda} ;
$$$$
g\left(\lambda, \xi_{0}, \xi_{e}\right)=\sum_{m=1}^{\infty} \frac{(4 m+1)\left(\xi_{0}+1\right) I_{2 m} P_{2 m}(0)}{2 m(2 m+1) \lambda \frac{Q_{2 m}\left(\xi_{0}\right) P_{2 m}\left(\xi_{e}\right)-P_{2 m}\left(\xi_{0}\right) Q_{2 m}\left(\xi_{e}\right)}{Q_{2 m}^{\prime}\left(\xi_{0}\right) P_{2 m}\left(\xi_{e}\right)-P_{2 m}^{\prime}\left(\xi_{0}\right) Q_{2 m}\left(\xi_{e}\right)}-\left(\xi_{0}+1\right)} ;
$$

$$
\begin{aligned}
& I_{2 m}=\int_{0}^{1} \ln (1+\eta) P_{2 m}(\eta) \mathrm{d} \eta, m=1,2, \ldots \ldots \\
& I_{2}=\frac{1}{12}, I_{4}=-\frac{3}{160}, I_{6}=\frac{5}{672}, I_{8}=-\frac{35}{9261}, \ldots \ldots .
\end{aligned}
$$

\section{Reference}

Batchelor, G. K. 1967 An Introduction to Fluid Dynamics. Cambridge Univ. Press, Cambridge, UK.

Chen, K. P., 2015 Fluid extraction from porous media by a slender semi prolate-spheroid: endeffect and shape optimization. Extreme Mech. Ltr. 4, 124-130.

Chen, K. P., Jin, Y. \& Chen, M. 2013 Pressure-gradient singularity and production enhancement for hydraulically-fractured wells, Geophysical J. Intl. 195, 923-931. 
Chu, J. Q., Jiao, W. P. \& Xu, J. J. 2008 Mathematical modelling study for water uptake of steadily growing plant root. Sci. China Ser. G-Phys. Mech. Astron., 51(2), 184-205.

Cinco-Ley, H. , Samaniego, V. F. \& Dominguez, A. N. 1978 Transient pressure behavior for a well with a finite-conductivity vertical fracture, SPE J.,18(4), 253-264.

Clausnitzer, V. \& Hopmans, J. V. 1994 Simultaneous modeling of transient three-dimensional root growth and soil water flow, Plant Soil, 164, 299-314.

Cowan, I. R. 1965 Transport of water in the soil-plant-atmosphere system, J. Appl. Ecol. 2, 221239.

Doussan, C., Vercambre, G. \& Pages, L. 1998 Modeling of the hydraulic architecture of root systems: an integrated approach to water absorption-distribution of axial and radial conductances in maize, Ann. Bot., 81, 225-232.

Frensch, J. \& Steudle, E., 1989 Axial and Radial Hydraulic Resistance to Roots of Maize (Zea mays L.), Plant Physiol., 91, 719-726.

Gardner, W. R. 1960 Dynamic aspects of water availability to plants, Soil Sci., 89, 63-73.

Hainsworth, J. M. \& Aylmore, L.A.G. 1986 Water extraction by single plant roots, Soil Sci. Soc. Am. J. 50, 841-848.

Jin, Y., Chen, K. P. \& Chen, M. 2015 Analytical solution and mechanisms of fluid production from hydraulically fractured wells with finite fracture conductivity. J. Eng. Math. 92, 103-122.

Landsberg, J. J. \& Fowkes, N. D. 1978 Water movement through plant roots, Ann. Bot., 42, 493508.

Melchior, W. \& Steudle, E. 1993 Water transport in onion roots. Plant Physiol., 101, 1305-1315. Michael, D.H. 1958 The separation of a viscous liquid at a straight edge, Mathematika 5, 82-84. 
Miles, J. W. 1965 Diffusion into a slender growing root. Proc. Roy. Soc. A, 284 (1396), 137-145.

Moffatt, H.K. 1964 Viscous and resistive eddies near a sharp corner, J. Fluid Mech. 18, 1-18.

Molz, F. J. 1981 Models of water transport in the soil-plant system, Water Resour. Res., 17, 1245-1260.

Passioura, J. B. 1988 Water Transport in and to roots, Ann. Rev. Plant Physiol. Plant Mol. Biol. 39, $245-265$.

Philip, J. R. 1957 The physical principles of soil water movement during the irrigation cycle, In: Proc. Intern. Congr. Irrigation and Drainage, $3^{\text {rd }}$, San Francisco, 8,125-154.

Phillips, O. M. 1991 Flow and Reactions in Permeable Rocks. Cambridge Univ. Press, Cambridge, UK.

Ratts, P.A.C. 2007 Uptake of water from soils by plant roots, Transp. Porous Med. 68, 5-28.

Richardson, S. 1970 A stickslip problem related to the motion of a free jet at low Reynolds numbers, Proc. Camb. Phil. Soc. 67, 477-489.

Roose, T. \& Fowler, A. C. 2004 A model for water uptake by plant roots, J. Theor. Bio. 228, 155-177.

Roose, T. \& Schnepf, A. 2008 Mathematical models for plant-soil interaction, Phil. Trans. $R$. Soc. A, 366, 4597-3611.

Smythe, W. R. 1989 Static and Dynamic Electricity, $3^{\text {rd }}$ Ed., Hemisphere Publishing, New York. Sprittles' J. E. \& Shikhmurzaev, Y. D. 2011 Viscous flows in corner regions: Singularities and hidden eigensolutions. International Journal for Numerical Methods in Fluids, 65, 372-382

Steudle, E. 2000 Water uptake by plant roots: an integration of views, Plant. Soil, 226, 45-56.

Steudle, E. \& Peterson, C. A. 1998 How does water get through roots? J. Experiment. Botany, 49, $775-788$. 
Stroock, A.D., Pagay, V. V., Zwieniecki, M. A. \& Holbrook, N. M. 2014 The Physicochemical Hydrodynamics of Vascular Plants. Ann. Rev. Fluid Mech.46, 615-642.

Trogdon, S.A. \& Joseph, D.D. 1980 The stick-slip problem for a round jet. 1. Large surface tension, Rheol. Acta. 19, 404-420.

van Genuchten (1980), M. Th. 1980 A closed form equation for predicting the hydraulic conductivity of unsaturated soils. Soil Sci. Soc. Am. J., 44, 892-898.

Zwieniecki, M. A., Thompson, M. V., and Holbrook, N. M. 2003 Understanding the Hydraulics of Porous Pipes: Tradeoffs Between Water Uptake and Root Length Utilization, J. Plant Growth Regul., 21, 315-323. 


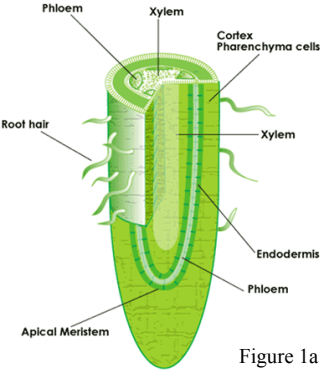




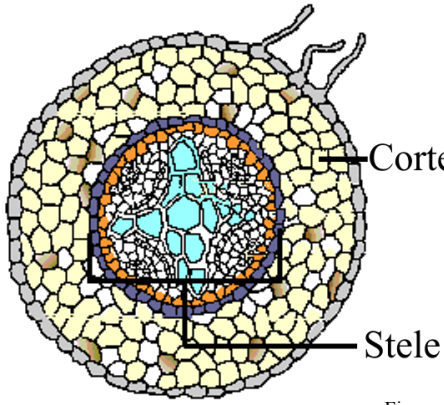

Figure $1 \mathrm{~b}$ 


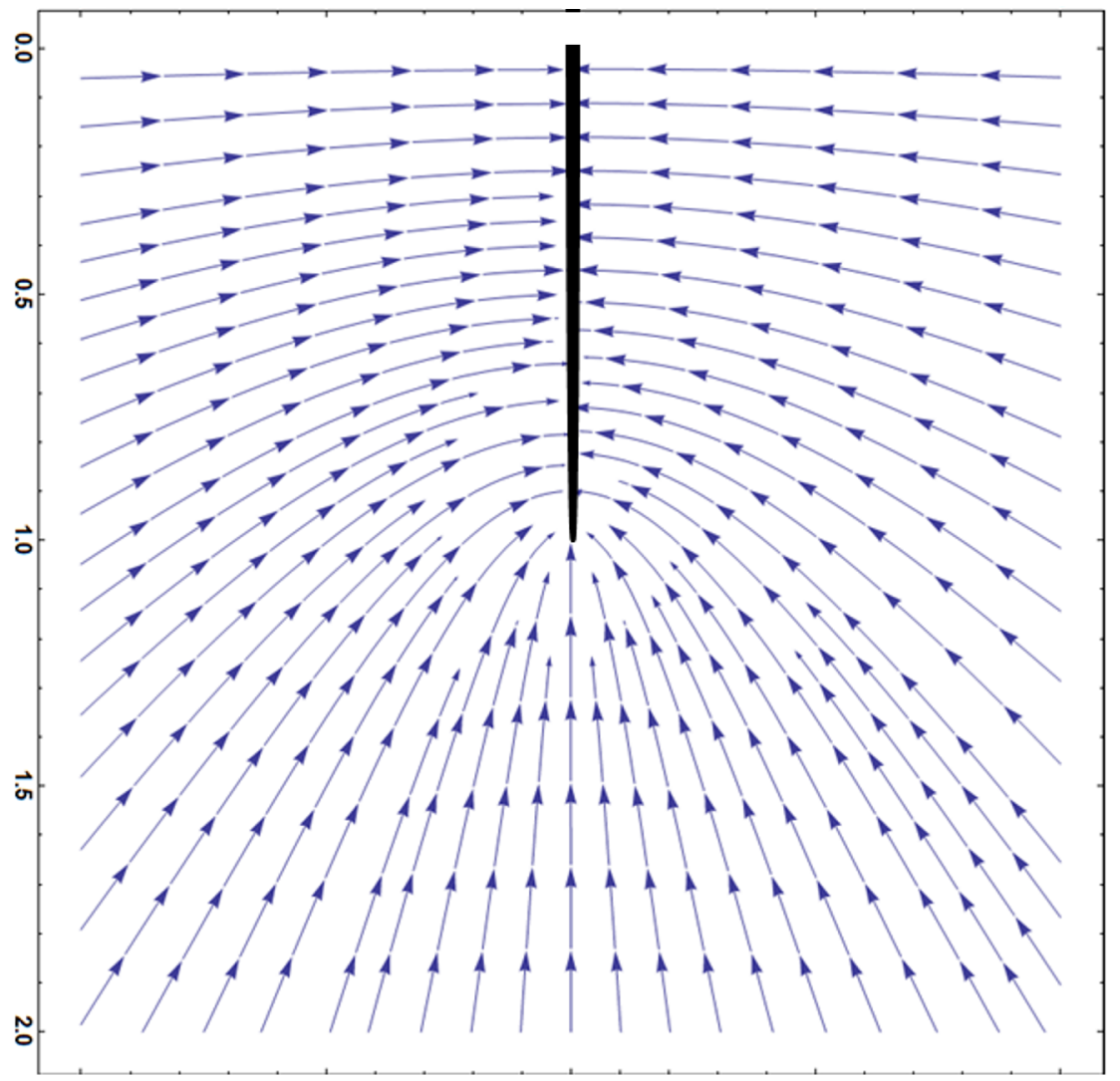

Figure 2 


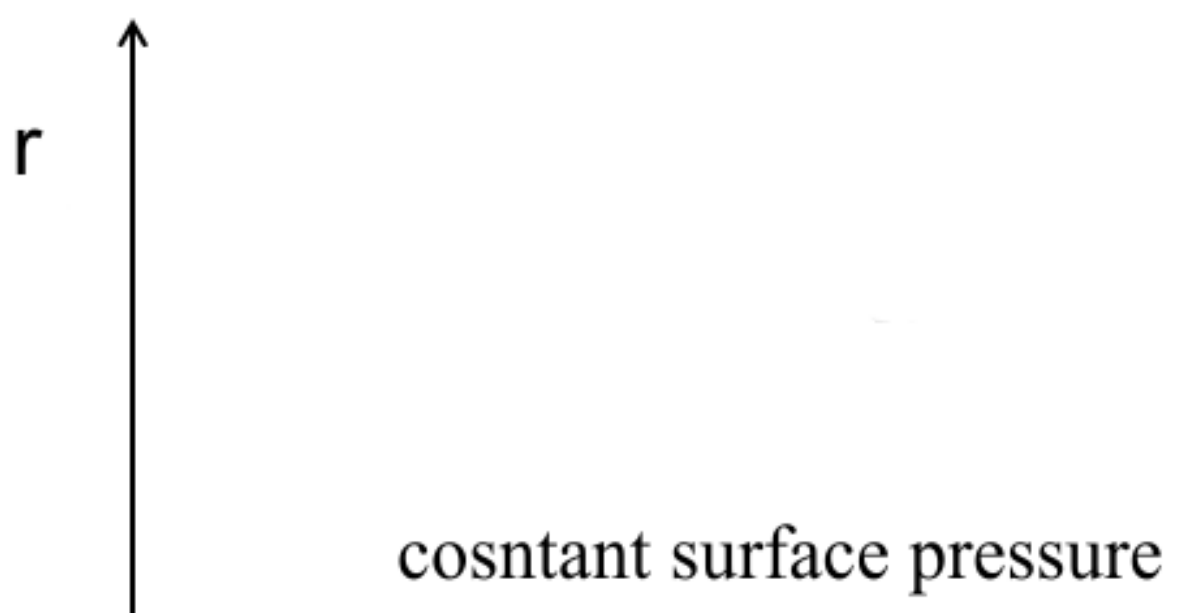

$\partial P / \partial n=0$

sealed root surface

cosntant surface pressure

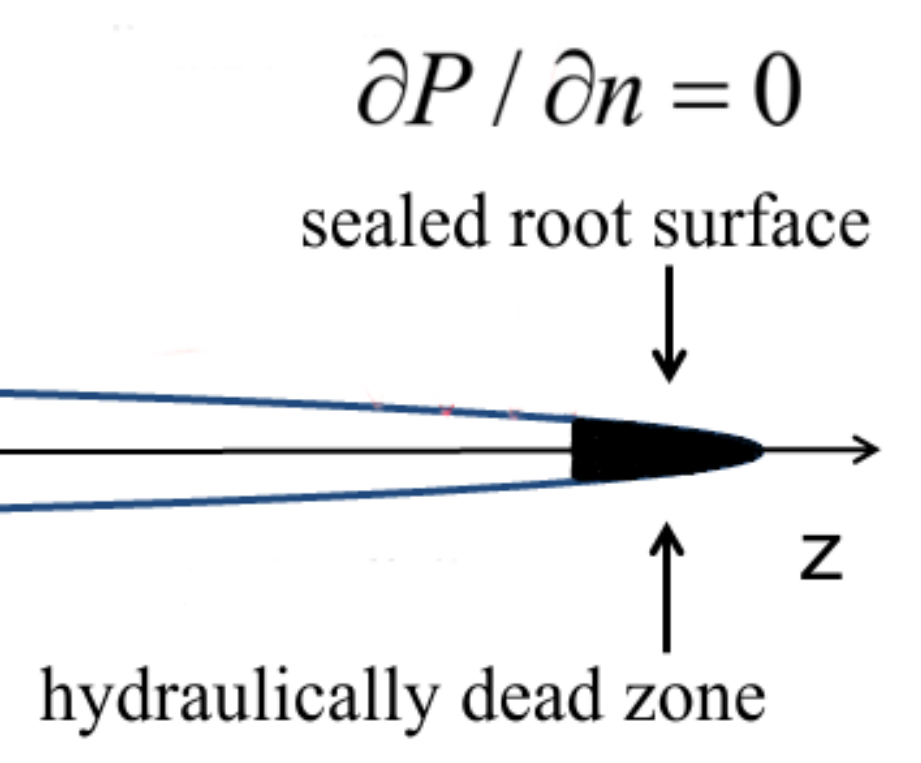


Figure 3b

Physical length of the root

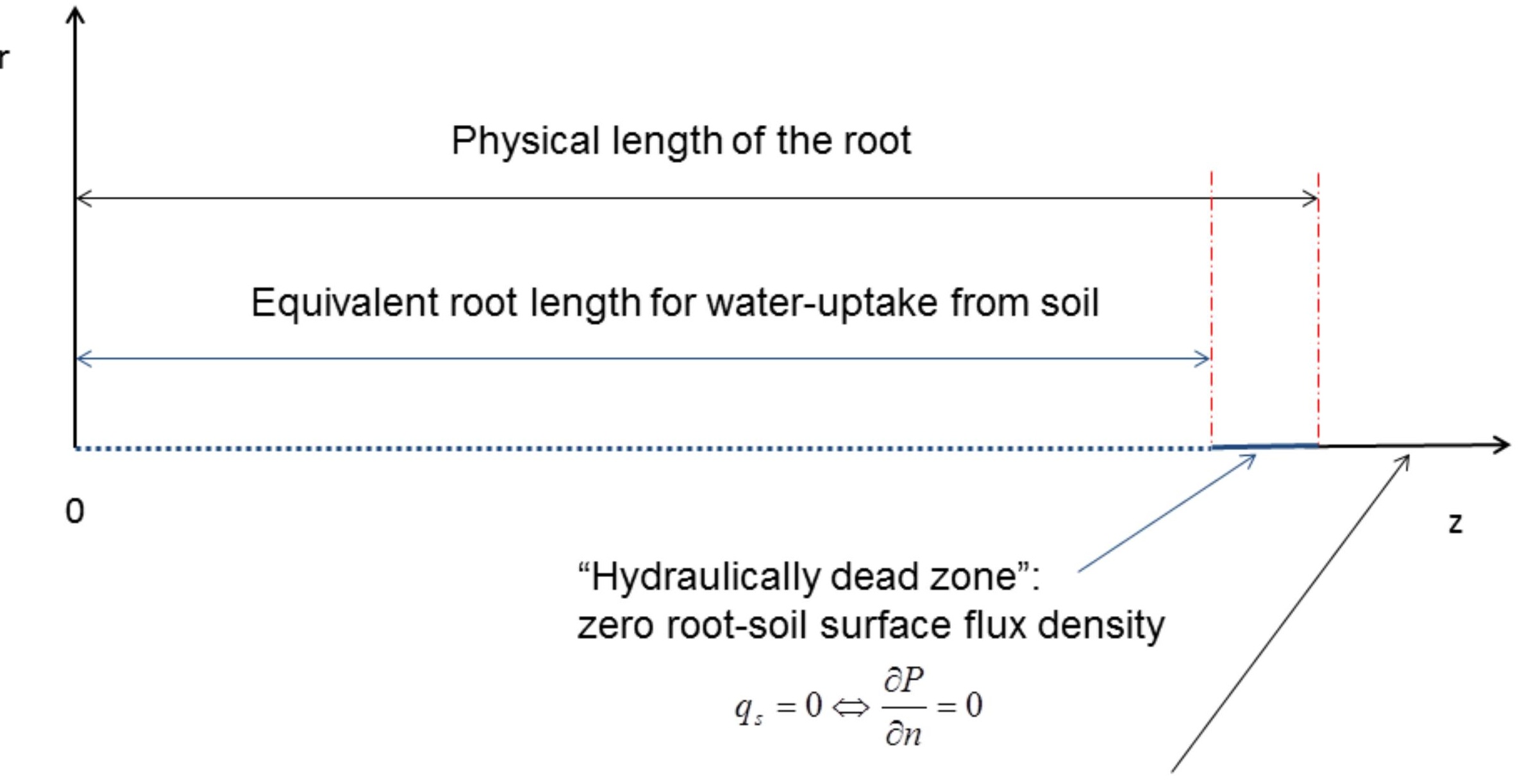

Symmetry about $\mathrm{x}$-axis $\quad \frac{\partial P}{\partial n}=0$ 
Root physical model

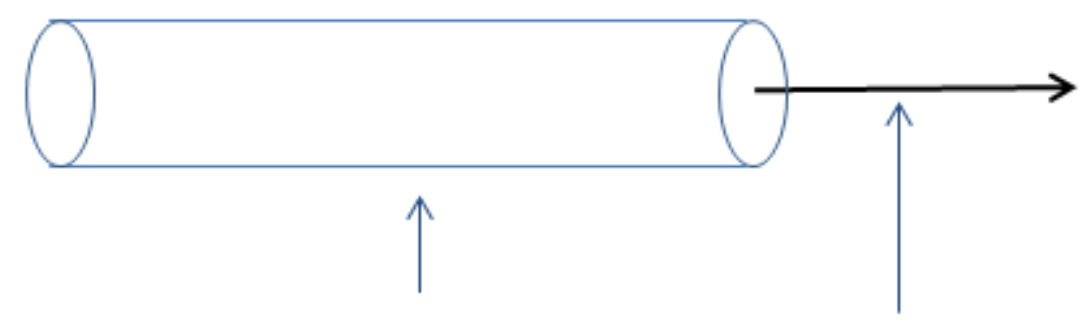

Porous concentric cylinder
Slender body

Symmetry

$$
\partial P / \partial n=0
$$

Root mathematical model

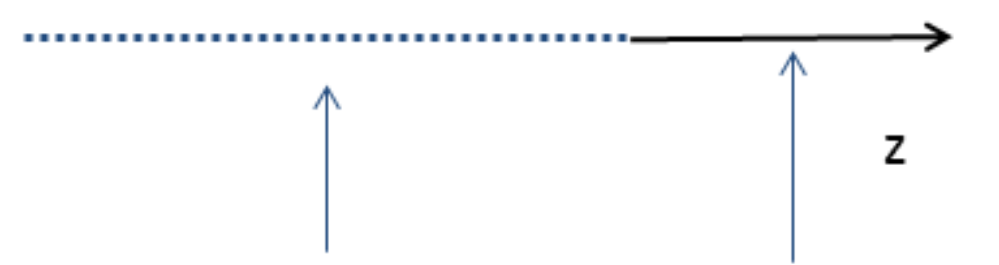

Line sink Symmetry $\partial P / \partial n=0$
Slender body

Symmetry

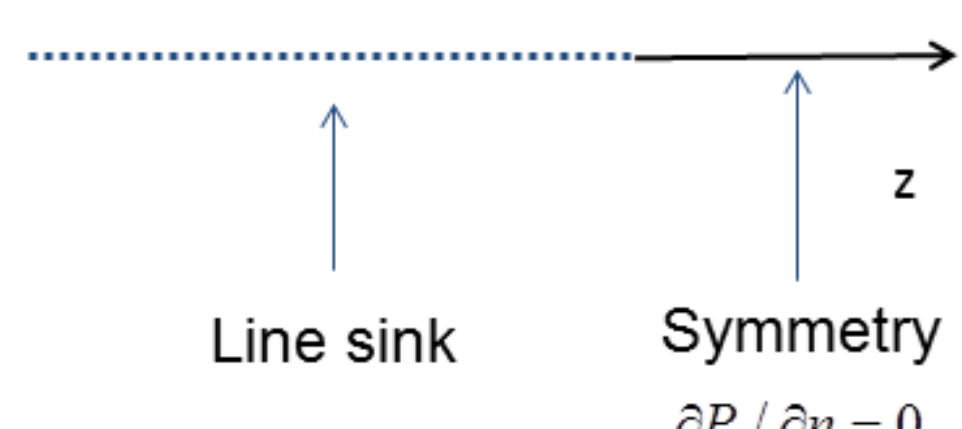

$$
\partial P / \partial n=0
$$


Figure 5

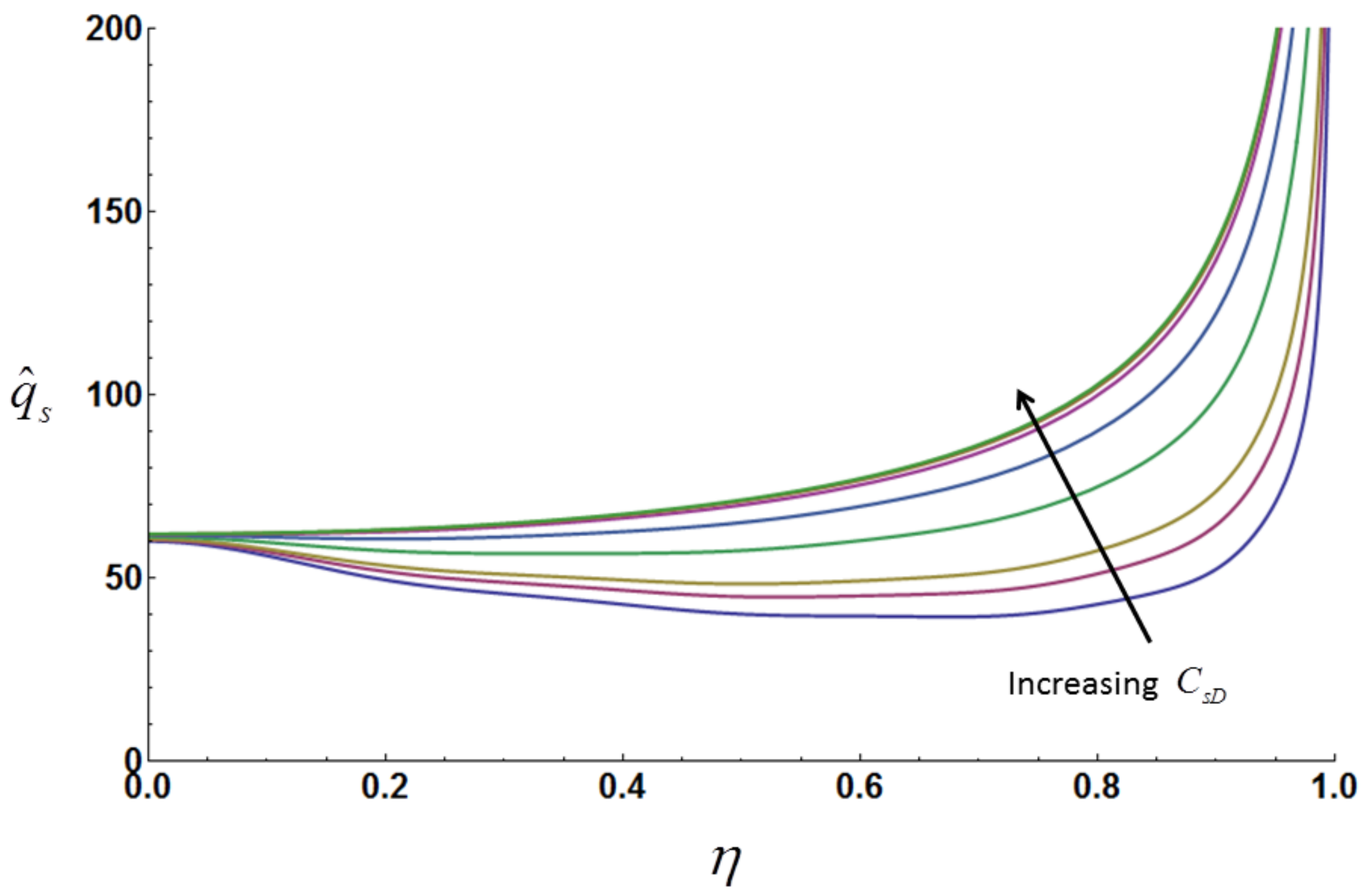


Figure 6

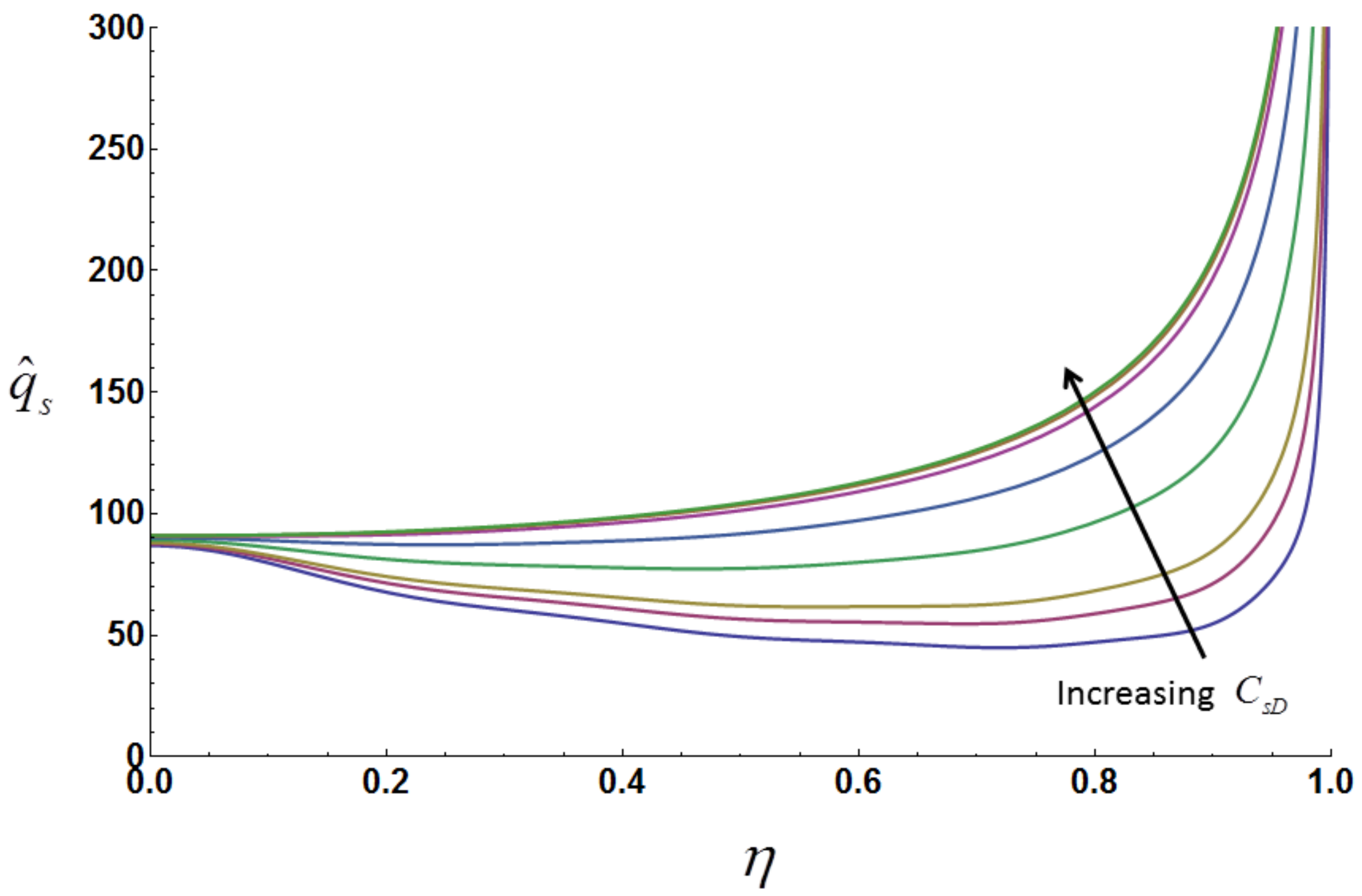


Figure 7

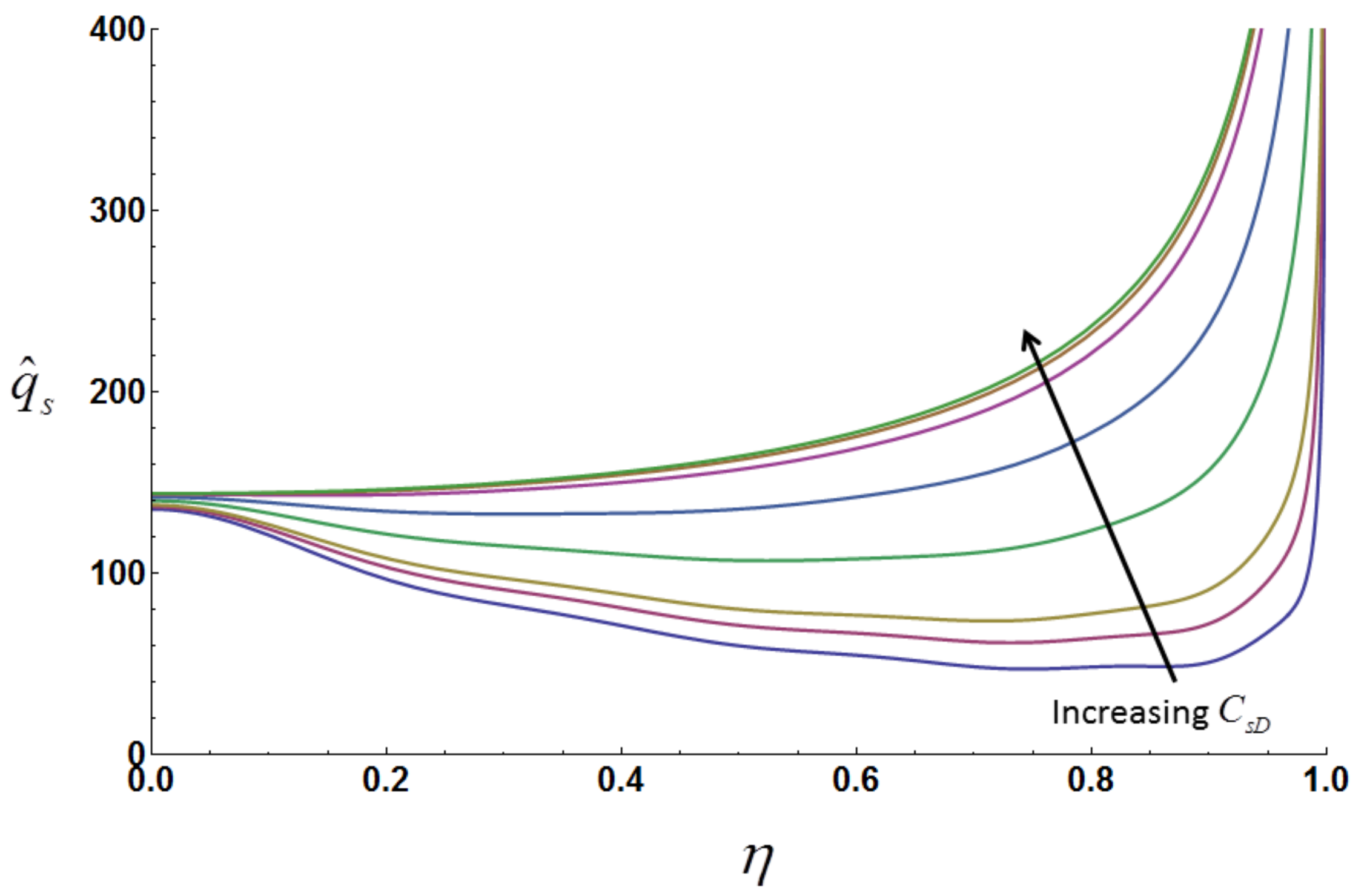


Figure 8

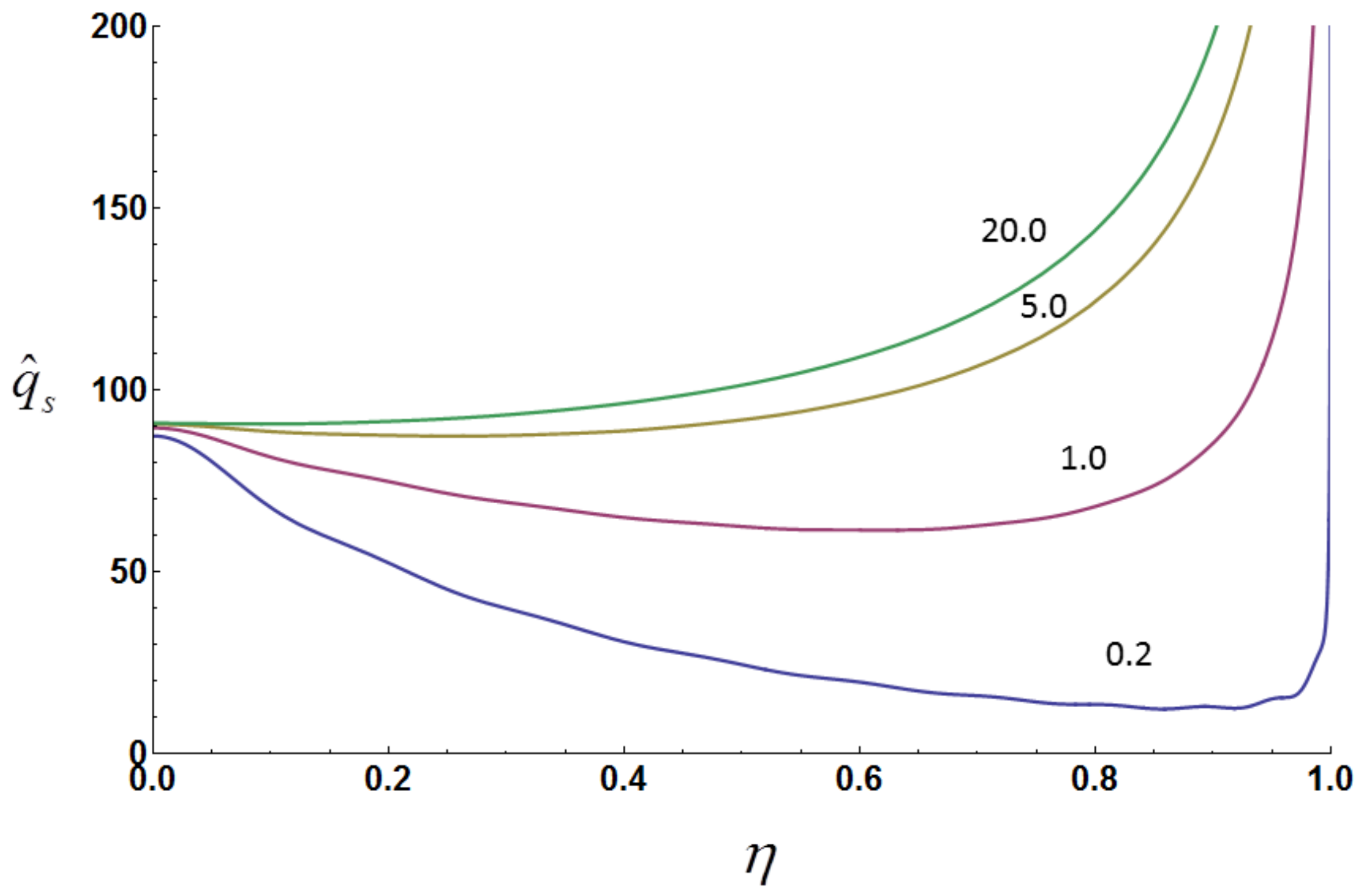


Figure 9

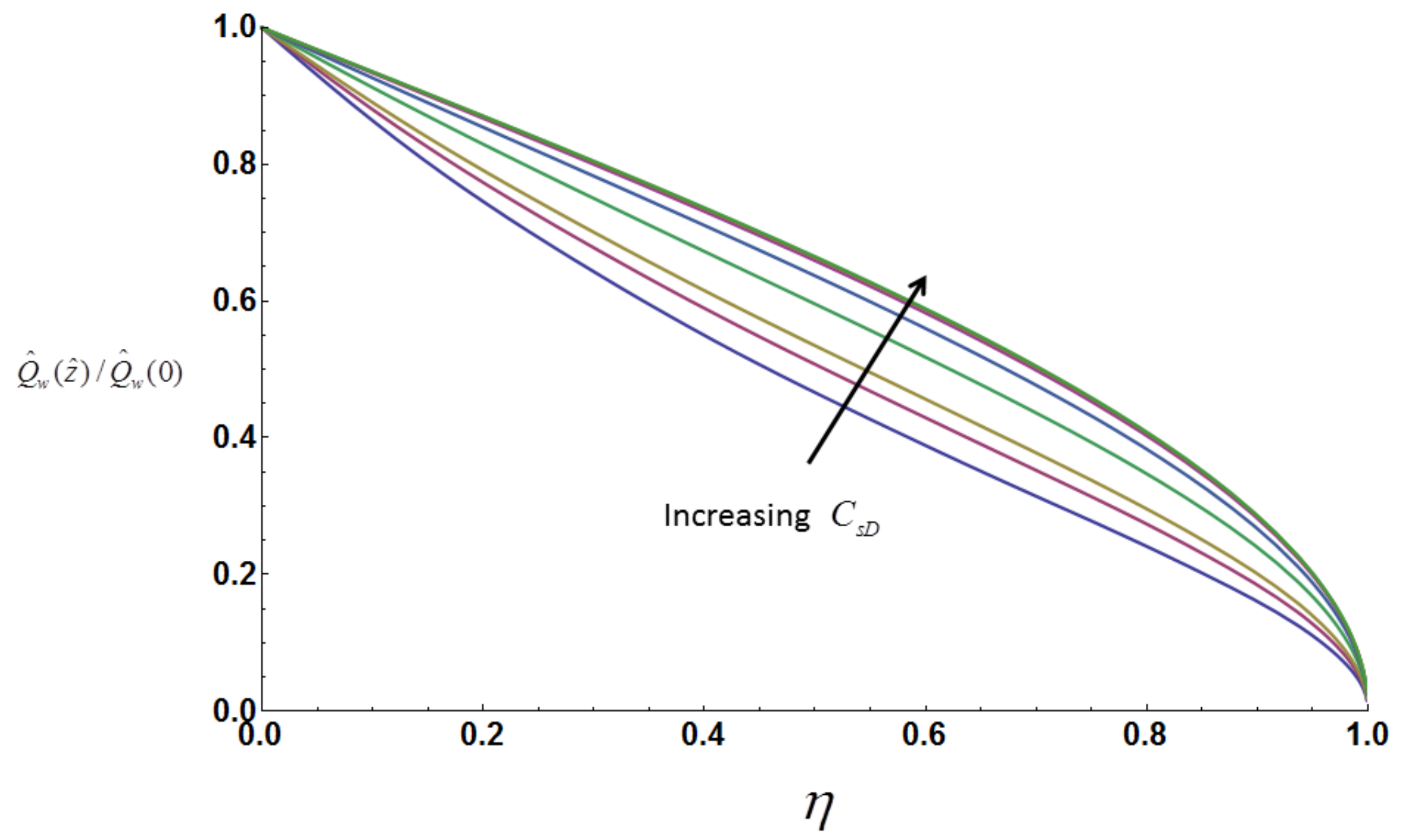


Figure 10

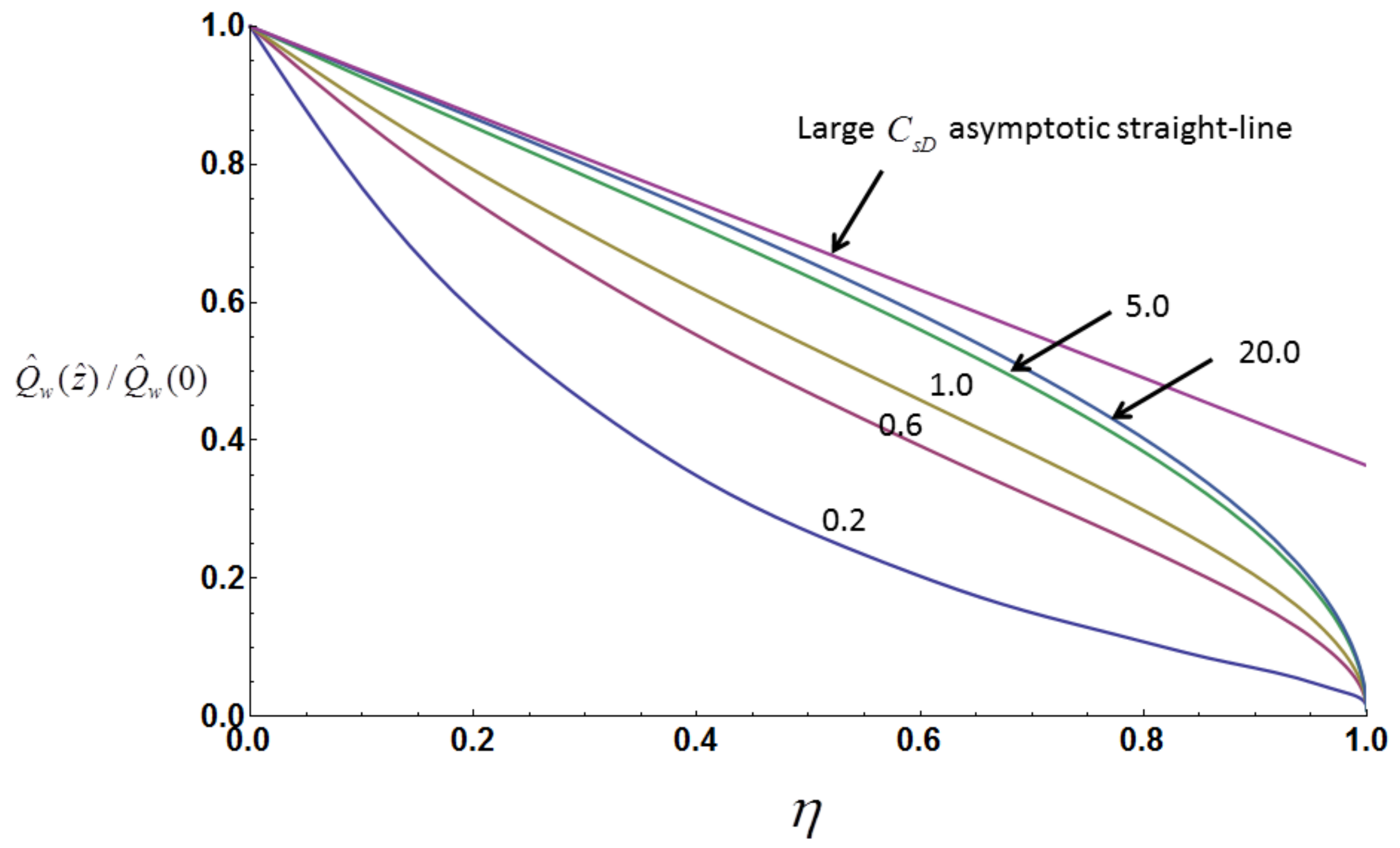


Figure 11

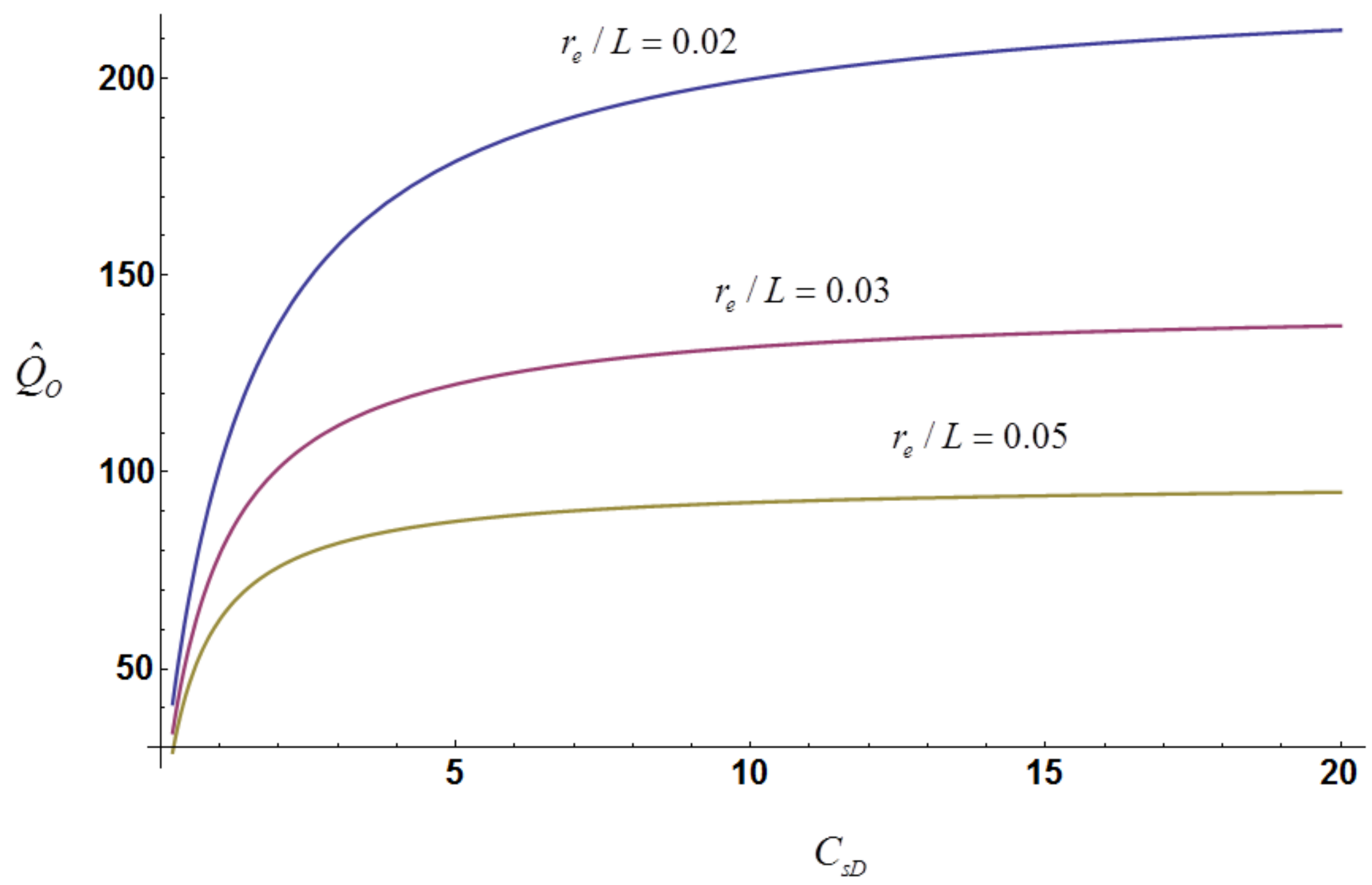

